\title{
1 Ecotoxicity of binary mixtures of - Microcystis aeruginosa and insecticides 3 to Daphnia pulex
}

4 Asselman $\mathrm{J}^{1,3}$, Janssen $\mathrm{CR}^{1}$, Smagghe $\mathrm{G}^{2}$, De Schamphelaere $\mathrm{KAC}^{1}$

$5{ }^{1}$ Laboratory of Environmental Toxicology and Aquatic Ecology, Ghent University, Belgium

6 'Laboratory of Agrozoology, Ghent University, Belgium

$7 \quad{ }^{3}$ Corresponding Author

8 Contact Information:

9 Email: jana.asselman@ugent.be

10 Postal Address: J. Plateaustraat 22, B-9000 Gent, Belgium

11 Tel: +3292643710

12 Fax: +32 92643766

13

14 


\section{$15 \quad$ Abstract}

16 In aquatic ecosystems, mixtures of chemical and natural stressors can occur which may significantly complicate risk 17 assessment approaches. Here, we show that effects of binary combinations of four different insecticides and 18 Microcystis aeruginosa, a toxic cyanobacteria, on Daphnia pulex exhibited distinct interaction patterns. 19 Combinations with chlorpyrifos and tetradifon caused non-interactive effects, tebufenpyrad caused an antagonistic 20 interaction and fenoyxcarb yielded patterns that depended on the reference model used (i.e. synergistic with 21 independent action, additive with concentration addition). Our results demonstrate that interactive effects cannot 22 be generalised across different insecticides, not even for those targeting the same biological pathway (i.e. 23 tebufenpyrad and tetradifon both target oxidative phosphorylation). Also, the concentration addition reference model provided conservative predictions of effects in all investigated combinations for risk assessment. These predictions could, in absence of a full mechanistic understanding, provide a meaningful solution for managing water quality in systems impacted by both insecticides and cyanobacterial blooms.

Capsule: Interactive effects between insecticides and cyanobacterial stressors cannot be generalized, not even for insecticides with closely related known modes of action.

Key words: mixture toxicity, insecticides, Microcystis aeruginosa, Daphnia 
In aquatic ecosystems, organisms are commonly exposed to mixtures of both natural and chemical stressors, resulting in potentially complex combined and interactive effects of natural and chemical stressors (Moe et al., 2013). This may significantly complicate risk assessment approaches of chemicals (Moe et al., 2013), which are currently still primarily focussed on a chemical-by-chemical basis (Landis et al., 2012; van Gestel et al, 2010). Consequently, there is a need for additional knowledge concerning these interactive effects to improve current risks assessment practices. Indeed, without taking interactive effects into consideration, predicted effects based on single substance datasets may over- or underestimate the effects actually occurring in the environment (Moe et al., 41 2013; van Gestel et al., 2010). Interactive effects may pose a significant threat to aquatic ecosystems as Holmstrup et al. (2010) currently reported synergistic interaction in over $50 \%$ of the 150 studies they reviewed focussing on interactive effects between chemical and natural stressors. However, the majority of these studies focussed on interactive effects of chemicals with abiotic stressors such as heat and oxygen depletion. Although some studies were identified that focussed on mixtures of chemicals and pathogens or parasites, Holmstrup et al. (2010) did not list any study relating to mixtures of chemicals and biotic stressors. The same conclusion can be drawn from the review by Laskowski et al. (2010), who listed only combinations of chemicals with abiotic natural stressors.

Of particular concern to zooplankton are cyanobacteria as they can outcompete other algae that normally serve as a food source to zooplankton (Moe et al., 2013: case study No.1 and Figure 2A). To date, five studies have investigated interactive effects of cyanobacteria and chemical stressors on zooplankton, mainly focussed on Daphnia as it is an extensively used model organism in risk assessment (OECD, 1998). Asselman et al. (2013), Cerbin et al. (2010) and Daam et al. (2011) studied interactive effects of cyanobacteria and pesticides. Bernatowicz and Pijanowska (2011) on the other hand studied interactive effects of cyanobacteria and PCBs while De Coninck et al. (2013) studied combinations of cyanobacteria and cadmium. Cerbin et al. (2010) and Daam et al. (2011) observed synergistic effects while Asselman et al. (2013) observed primarily antagonistic effects. Bernatowicz and Pijanowska (2011) observed interactive effects that differed depending on the genotype of Daphnia while De Coninck et al. (2013) did not on average observe interactive effects for the different genotypes studied. Despite this relatively 
small amount of available studies and although methods and reference models vary, a complex pattern of interactive effects between cyanobacteria and chemicals is emerging and therefore this requires further study with a consistent set of reference models.

Here, we will specifically focus on the combined effects of insecticides and cyanobacteria. Indeed, these interactive effects are of particular concern for aquatic ecosystems, as cyanobacteria often proliferate under conditions favouring eutrophication, which are conditions often also associated with pesticide pollution (Moe et al., 2013; Daam et al., 2011). In addition, Moe et al. (2013) noted that pesticides may affect zooplankton leading to a decreased grazing pressure and as a result a potential increase in phytoplankton density, including cyanobacteria. Furthermore, Cerbin et al. (2010) observed synergistic effects for combination of Microcystis aeruginosa and carbaryl whereas Asselman et al. (2013) observed non-interactive and antagonistic effects for combinations of carbaryl with 4 species of cyanobacteria (Aphanizomenon sp., Cylindrospermopsis raciborskii, Microcystis aeruginosa and Oscillatoria sp) albeit on different endpoints. As these studies have focused solely on the insecticide carbaryl, it is difficult to generalize these results to other insecticides.

Thus, the first goal was to study the interactive effects between insecticides and cyanobacteria by focusing on a broader range of insecticides with different molecular targets and to investigate to what extent results can be extrapolated across other molecular targets of other chemicals. We combined these insecticides with Microcystis aeruginosa, one of the most common and most toxic cyanobacteria in eutrophic freshwater ecosystems (Carmichael, 1995). This small circular, bloom-forming cyanobacterium is well-known to produce several toxins such as microcystins (van Apeldoorn et al., 2007). The effects of $M$. aeruginosa on the aquatic system and Daphnia species in particular have been extensively studied (Asselman et al., 2012; Lürling, 2003; Rohrlack et al., 1999). Yet, the exact mechanisms through which $M$. aeruginosa affects zooplankton species remain unclear. Three different factors have been put forward in literature: the production of toxins, the presence of feeding deterrents and the lack of essential nutrients compared to green algae (DeMott et al., 2001; Lürling, 2003).

We exposed Daphnia pulex for 21 days to binary combinations of $M$. aeruginosa and four insecticides. We specifically selected chlorpyrifos, fenoxycarb, tebufenpyrad and tetradifon as these insecticides span a wide range 
of molecular targets (IRAC, 2009) to study whether interactive effects occur across molecular targets. Chlorpyrifos, an organophosphate, was specifically selected as it inhibits acetylcholine esterase and its molecular target is therefore similar to carbaryl, which is a carbamate also inhibiting acetylcholine esterase (IRAC, 2009), although the exact molecular mechanism of the inhibition is not completely identical. In particular, the formation of the enzyme inhibiting complex between the insecticide and acetylcholine esterase is identical for both chlorpyifos and carbaryl (Fukuto, 1990). However, the recovery half-life of the enzyme, i.e. regeneration of the enzyme, is much smaller for carbaryl than for chlorpyrifos (Fukuto, 1990). In contrast, fenoxycarb, is a juvenile hormone mimic and intereferes with cellular processes involving juvenile hormones (IRAC, 2009). The other two insecticides, tebufenpyrad and tetradifon both affect the oxidative phosphorylation albeit through different mechanisms (IRAC, 2009). Tebufenpyrad is a mitochondrial complex I electron transport inhibitor; while tetradifon inhibits the ATP-synthase (IRAC, 2009).

Also, it has been shown that toxicants, affecting the same biological pathways or processes, while not interacting with the same molecular target, can cause interactive effects on the exposed organism (Deneer, 2000; Van Gestel et al., 2011). The second goal of this research was to add knowledge concerning the interactive effects of mixtures, with the a priori knowledge about the molecular targets of the insecticides allowing us to formulate hypotheses about the occurrence of interactive effects between these stressors. In doing so, we expected to gain knowledge about the importance of molecular targets and shared common pathways in interactive effects.

Given the four studied insecticides, we can put forward three distinct hypothesis. Chlorpyrifos is very similar to carbaryl so we hypothesize that the interactive effects with $M$. aerugonisa would be similar, i.e. antagonistic (Asselman et al., 2013) and can be extrapolated from one insecticide to another. Fenoxycarb does not have the same molecular target as carbaryl nor does it affect any of the biological pathways affected by M. aeruginosa and consequently no interactive effects were expected. In contrast, tebufenpyrad and tetradifon may potentially cause interactive effects when combined with $M$. aeruginosa as they all affect the oxidative phosphorylation pathway albeit through different molecular mechanisms (Asselman et al., 2012). Thus in summary, based on knowledge and 
available literature concerning molecular targets and affected biological pathways, we hypothesize that antagonistic or non-interactive effects can be observed for chlorpyrifos and fenoxycarb whereas synergistic effects can be observed for tetradifon and tebufenpyrad, when combined with M. aeruginosa. Even though the exact mechanism through which $M$. aeruginosa affects Daphnia is unknown, it is fair to assume the same mechanism will be operating across the four experiments with the four insecticides, as the same Daphnia clone will be used as well as the same concentration of $M$. aeruginosa.

\section{Material and Methods}

\section{Animals}

Animals originated from isolates obtained from the laboratory of JR Shaw (School of Public and Environmental Affairs, Bloomington, IN, USA; Shaw et al., 2007). Animals were grown in maintenance cultures synchronized in age. Cultures were maintained in no nitrogen, no phosphorous COMBO medium (Shaw et al., 2007, Kilham et al., 1998) under constant environmental conditions $\left(20 \pm 1{ }^{\circ} \mathrm{C}, 16: 8\right.$ light-dark; light intensity: $14 \mu \mathrm{mol}$ photon $\left.\mathrm{m}^{2}{ }^{2} \mathrm{~s}-1\right)$. Food consisted of a mixture of green algae, i.e. Pseudokirchneriella subcapitata and Chlamydomonas reinhardtii, in a 3 to neonates ( $<24 \mathrm{~h}$ old) that were used in all the experiments.

\section{Cyanobacteria}

Microcystis aeruginosa strain (PCC7806) was obtained from the Pasteur Culture Collection of Cyanobacteria (Institut Pasteur, Paris, France). Prior to use in experiments, M. aeruginosa was grown in batch in a referenced medium (Institut Pasteur; Stanier et al., 1971) at $20 \pm 1^{\circ} \mathrm{C}$ under constant light intensity $\left(14 \mu \mathrm{mol}\right.$ photon $\left.\mathrm{m}-{ }^{2} \mathrm{~s}-1\right)$ with gentle aeration. After ten days, the cells were harvested, centrifuged to remove excess medium and counted under a light microscope as well as an automated Coulter Counter. In addition, the dry cell weight was determined.

\section{Chemical Stock Solutions}



purity $\geq 99 \%$ ). Due to low water solubility of the compounds, stock solutions were made by dissolving the insecticides in pure ethanol (analytical grade, Sigma-Aldrich, Bornem, Belgium) .

\section{Chronic Toxicity Experiments}

The experimental design in the chronic toxicity experiments was identical to Asselman et al. (2013). Briefly, the central composite design was used with the addition of five points for each of the two single stressors (Fig. 1A-D). This allowed simultaneous evaluation of the effects of the individual stressors and the mixtures as recommended by De Laender et al (2009). Furthermore, the central composite design (Fig. S1) has been identified as one of the better designs to evaluate mixture toxicity with the currently available models (Jonker et al., 2005). Each designpoint was replicated three times, i.e. three daphnids per treatment. A solvent control was added to exclude potential effects of the solvent. Control and solvent control were replicated five times. Concentration ranges of the single treatments were determined based upon literature review and preliminary experiments with the single compounds. They were selected in such a manner to cover the range of different EC50s (effect concentration causing a 50\% decline in reproduction) reported in literature and observed in our preliminary experiments.

The experiments were conducted in $25 \mathrm{~mL}$ glass vessels, containing a single neonate each, and lasted for 21 days. During the experiments, medium was renewed every two days. Animals were fed daily. In treatments containing M. aeruginosa, animals were given $M$. aeruginosa as a percentage ranging from $5 \%$ to $80 \%$ of the diet (on a dry weight basis, i.e. 0.1 to $1.6 \mathrm{mg}$ dry weight) depending on the treatment. Additional green algae were supplemented to each of these treatments. Therefore, each treatment always received a total of $100 \%$ food per day based on dry weight per litre (i.e. $2 \mathrm{mg}$ dry weight of food per liter per day). 

concentrations and not nominal concentrations.

\section{Data analysis}


The chosen response variable to determine mixture toxicity was total number of offspring per surviving female at

177

178

179

the end of the experiment. Females that did not survive were excluded from the analysis. In tebufenpyrad and tetradifon treatments, animals in the highest concentration, $25 \mu \mathrm{g} / \mathrm{L}$ and $40 \mu \mathrm{g} / \mathrm{L}$ respectively, all died within the first week. Therefore, these concentrations were not taken into account in data analysis or in the concentration measurements. First, results from control treatments and solvent control treatments were analysed and compared to exclude solvent effects. Comparison was done using a t-test after verifying assumptions of normality and homoscedasticity by the Shapiro-Wilk test (Royston, 1982) and Levene test (Fox, 2008), respectively.

The analysis of the binary mixture experiments was conducted according to the R-code from Asselman et al. (2013) (R Development Core Team, 2008). This code evaluates mixture toxicity experiments with both the concentration addition and the independent action reference models based on Jonker et al. (2005). It uses the log logistic dose response function (eq. 1) and subsequent derived equations for both concentration addition (eq. 2) and independent action (eq. 3) reference model as described by Jonker et al. (2005) and listed in the R-code provided by Asselman et al. (2013), which is accessible through their supplementary information (http://onlinelibrary.wiley.com/doi/10.1002/etc.2296/suppinfo).

The log-logistic response function is defined as follows:

$$
f=y=\frac{k}{1+\left(\frac{x}{x_{50}}\right)^{s}} \quad \text { (eq. 1) }
$$

In which $\mathrm{y}$ is the response variable (total reproduction), $\mathrm{k}$ is the value of the response variable at $\mathrm{x}=0, \mathrm{x}$ is the concentration of the insecticide or the $\%$ of $M$. aeruginosa in the diet, $s$ is the slope parameter and $x_{50}$ is the concentration of the insecticide or the $\%$ of $M$. aeruginosa in the diet resulting in a decline of the response variable of $50 \%$ compared to $\mathrm{k}$ (Jonker et al., 2005).

In a mixture, under the concentration addition reference model, the sum of the toxic units is assumed to equal 1 in case of no interaction and this is expressed as follows:

$$
\frac{x_{1}}{f_{1}^{-1}(y)}+\frac{x_{2}}{f_{2}^{-1}(y)}=\exp (G)(\text { eq. 2) }
$$


Here, indexes 1 and 2 denote stressors 1 and 2 in the mixture and $f^{-1}$ denotes the inverse of the response function as in eq. $1, \mathrm{x}$ is the concentration of the stressors. G refers to the deviation function (see eq. 4 , as defined in Jonker et al., 2005) and the value of $\mathrm{G}$ equals 0 under the hypothesis of no interaction.

Under the independent action reference model, the response to stressor 1 is assumed to act independently from stressor 2 and this is mathematically expressed as follows:

$$
y=k \phi\left(\phi^{-1}\left[k\left(\frac{1}{1+\left(\frac{x_{1}}{x_{50}}\right)^{s_{1}}}\right)\left(\frac{1}{1+\left(\frac{x_{2}}{x_{50_{2}}}\right)^{s_{2}}}\right)\right]+G\right) \text { (eq. 3) }
$$

In which indexes 1 and 2 again denote stressor 1 and 2 in the mixture and $\phi$ refers to the cumulative standard normal distribution function. All other parameters are identical to those in equations 1 and 2.

The deviation function $\mathrm{G}$ is defined identically for both reference models and is as follows (Jonker et al., 2005):

$$
G=\exp \left(a\left(\frac{\frac{x_{1}}{x_{501}}}{\frac{x_{1}}{x_{501}}+\frac{x_{2}}{x_{502}}}\right)\left(\frac{\frac{x_{2}}{x_{502}}}{\frac{x_{1}}{x_{501}}+\frac{x_{2}}{x_{502}}}\right)\right)(\text { eq. 4) }
$$

In which a is the parameter that quantifies deviations from the reference model. All other parameters are identical to the ones in equations 1, 2 and 3 (From Jonker et al., 2005). The more positive the deviation parameter, the more antagonistic the mixture deviates from the reference model. The more negative the deviation parameter, the more synergistic the mixture deviates from the reference model.

Briefly, we analysed the data in three steps. Step one fits the reference model (IA or CA) to the data from individual stressor treatments only and not from the mixture treatments (i.e. equation 2 or 3 in which $\mathrm{G}=0$, because the parameter $a$ is not included in this step and is therefore set to zero in equation 4, i.e. no interaction) . Step two then fits the reference model (IA or CA) to all the data from all individual and all mixture stressors treatments (i.e. equation 2 or 3 in which $\mathrm{G}=0$ because the parameter $\mathrm{a}$ is not included in the model yet and is therefore set to zero in equation 4, i.e. still no interaction). In step three the reference model (IA or CA) is extended with the deviation parameter a to quantify deviations from non-interaction (i.e. equation 2 or 3 in which $\mathrm{G}$ is not zero anymore as the parameter $\mathrm{a}$ is added to the model based on equation 4).The significance of the addition of the deviation parameter to the reference model is statistically determined through an F-statistic (Asselman et al., 2013; Jonker et al., 2005). Alternatively the Akaike Information Criterion (AIC) can be used to give an indication of the best model fit although it is not a true statistical measure. To exclude the possibility that a single design point would affect 
conclusions, models of steps two and three were subsequently fitted and analysed by each time leaving out one design point. No design point significantly influenced statistical conclusions as leaving one design point out did not alter the p-value (Table S1).

\section{Results}

Measured concentrations of insecticides are reported (Fig. 1A-D). In all experiments, effects of the solvent carrier were negligible as organisms in solvent controls reproduced comparably to organisms in controls ( $p=0.57)$ (Fig S2S5). Mortality in control treatments was less than $10 \%$ for all experiments (Fig. S2-S5). Across all experiments in all treatments, $\mathrm{pH}$ differed at most 0.25 units from control $\mathrm{pH}$ (COMBO medium $\mathrm{pH}=7.5)$. The effect concentration for the total reproduction per surviving female resulting in a $50 \%$ decline in of this total reproduction (EC50) was for all insecticides within the tested concentration range as can be seen from the single dose response data (Fig. S2-S5) and the model estimates of step 1, using only single stressor data (Table 1). Fenoxycarb and chlorpyrifos were the most toxic, both having an EC50 around $70 \mathrm{ng} / \mathrm{L}$ whereas tetradifon and tebufenpyrad were significantly less toxic with EC50s around $10 \mu \mathrm{g} / \mathrm{L}$ (Table 1).. The estimated EC50 for M. aeruginosa ranged from $30 \%$ to $60 \%$ of the total diet between experiments (Table 1 Fig. S6-S9). In the experiments with fenoxycarb and tetradifon (Fig. S7 and S9), animals exposed to $80 \%$ of $M$. aeruginosa either died or survived without reproducing whereas in experiments with chlorpyrifos and tebufenpyrad (Fig. S6 and S8), animals exposed to $80 \%$ of $M$. aeruginosa were able to reproduce, albeit very little. In the mixture treatments, animals did not survive in combination of chlorpyrifos and $M$. aeruginosa containing the highest chlorpyrifos concentration (Fig. S10) In the three other combinations, at least one animal survived in each mixture combination (Fig. S11-S12).

A first indication of combined effects of the binary mixtures is represented in Fig. 2 (left panel A-D). Here, predictions of the mixture data were made with the reference models based upon the parameters as estimated by reference models based upon the single stressor data only (step 1, see Materials and Methods for details). From these predictions, it indicates no clear interactive effects for chlorpyrifos, fenoxycarb and tetradifon. Indeed, in figure $2 \mathrm{~A}, 2 \mathrm{~B}$ and $2 \mathrm{D}$ both single stressor data points (open circles and triangles) and mixture data points (filled circles and triangles) lie close to the 1:1 line which means that the fitted (single data points) and predicted (mixture 
points) values closely match the observed values. In contrast, for tebufenpyrad (Fig. 2C), the mixture points all lie below the 1:1 line and there predicted values are quite different from the observed values. This indicates an antagonistic effect as observed reproduction is larger than reproduction predicted with the CA and IA reference models.

Fitting both the CA and IA reference models to the entire dataset Fig. 2 (middle panel E-H), i.e. step2, results in similar conclusions as the ones obtained from step1 (i.e. model fit to only the single stressor data), i.e. no clear interactions as all observations lie close to the 1:1 line for chlorpyrifos, fenoxycarb and tetradifon (Fig. 2E, 2F and $2 \mathrm{H}$ ). For tebufenpyrad (Fig. $2 \mathrm{G}$ ), the mixture points now fit better as they lie closer to the $1: 1$ line but the single points have moved further from the 1:1 line indicating this is not a good model fit. Extending the reference models with the deviation parameter a (Eq. 4) does not improve the results clearly for chlorpyrifos and tetradifon Fig. $2 \mathrm{I}$ and $2 \mathrm{~L}$. For fenoxycarb, i.e Fig. 2J, the fit becomes only slightly better as all points move closer to the 1:1 line. For tebufenpyrad, Fig. 2K, both single and mixture points are now closer to the 1:1 line than in Fig. $2 \mathrm{G}$.

Statistical comparisons between the reference model and the reference model extended with the deviation parameter revealed for two out of the four combinations (i.e. chlorpyrifos and tetradifon) no deviations from noninteraction based on either the concentration addition model or the independent action model (Table 1 , $p$ value $>0.05$ ). This means that for both mixtures the deviation parameter a is not significantly different from zero, making the deviation function $G$ (equation 4) zero in both the independent action (equation 3 ) as well as the concentration addition model (2). In contrast, the binary combination of tebufenpyrad and M. aeruginosa resulted in antagonistic effects on the reproduction of $D$. pulex based on both reference models (Table $1, p$-value $<0.05)$. The deviation parameter a for both concentration addition and independent action is around 2.5 , which indicates antagonistic deviation from the reference models. For binary combinations of fenoxycarb and $M$. aeruginosa a synergistic deviation was observed when analysed with the independent action model (Table $1, \mathrm{P}$-value<0.05). Analysis with the concentration addition model for this combination concluded non-interaction (Table 1, $\mathrm{p}$ value $>0.05)$, meaning the deviation parameter a becomes zero, resulting in the sum of toxic units equalling 1 in equation 2. The sum of squared errors (SSE) for the independent action reference model (618.4) was slightly higher than the SSE for the concentration addition model (536.9). However, addition of the deviation parameter to the 
independent action model reduced the SSE to 494.2, which is slightly lower than the SSE of the concentration addition reference model. AIC criteria were comparable between the best model based on concentration addition (268.58) and the best model based on independent action (268.441).

\section{Discussion}

Despite the increasing awareness of interactive effects between stressors in aquatic ecosystems, the knowledge about these potential interactions, their occurrence and intensity remain limited (Holmstrup et al., 2010; Moe et al., 2013). Yet, such knowledge is essential to adequately protect and conserve our ecosystems. Our study added to the growing body of knowledge on interactive effects between cyanobacteria and insecticides. Furthermore, where previous studies have used a variety of analysis methods such as generalized linear models, factorial regression and analysis of variance, our present study focussed on using standard reference models of mixture toxicity in a consistent manner. We observed different interactive effects for the different insecticides depending on the reference model used.

In contrast to the postulated hypothesis, combinations of chlorpyrifos and $M$. aeruginosa resulted in noninteractive effects and not in antagonistic effects as we observed earlier for combinations of carbaryl and $M$. aeruginosa with the two reference models (Asselman et al., 2013). Together, these two observations suggest that even for insecticides that target the same enzyme (i.e. acetycholine esterase), the observation of interactive or additive effects cannot be extrapolated from one insecticide to another. However, inhibition of acetylcholine esterase by organosphosphates is less reversible and hence longer-lasting than inhibition of acetylcholine esterase by carbamates (Pope et al., 2005). This difference in recovery time may be a potential explanation for the shift in combined effects with cyanobacteria from antagonistic interaction with carbaryl (a carbamates) to additivity with chlorpyrifos (an organophosphate). Alternatively, choline esterase inhibitors have been shown to target other molecules than the acetylcholine esterase (Pope et al., 2005). Differences in the ability to target other molecules could also be a potential explanation for the differences in interactive effects observed for carbaryl and chlorpyrifos. Likewise, differences in metabolic activation and degradation between organophosphates and carbamates (Fukuto, 1990) may also be a potential cause for the difference in interaction pattern with $M$. aeruginosa. 
Likewise, tebufenpyrad and tetradifon, both targeting the oxidative phosphorylation albeit through different molecular mechanisms, demonstrated different combined effects with Microcystis. Combinations with tebufenpyrad were antagonistic whereas combinations with tetradifon were additive. Tetradifon inhibits ATPsynthases while tebufenpyrad inhibits NADH : ubiquinone reductase activity in complex I of the mitochondrial respiration (IRAC, 2009; Sherer et al., 2006). These molecular targets correlate to some extent with results obtained by Asselman et al. (2012) when exposing D. pulex to M. aeruginosa. Asselman et al. (2012) observed upregulation of one ATP synthase gene out of the 6 ATP synthases and 11 NADH: ubiquinone oxidoreductase genes out of a total of 26 genes in the genome. Indeed, exposure to $M$. aeruginosa mainly stimulates NADH: ubiquinone oxidoreductases rather than ATP-synthases. As a consequence, it is more likely that tetradifon and $M$. aeruginosa do not act interactively as they each affect different parts of the oxidative phosphorylation. Of course, further mechanistic research is needed as RNA levels do not necessarily correlate with protein levels.

These observations, i.e. different interactive effects for insecticides with closely related molecular targets (e.g. carbaryl - chlorpyrifos, tetradifon - tebufenpyrad) when combined with the same stressor (here: $M$. aeruginosa) in a binary mixture, suggest a potential mechanistic basis for interactive effects that may well be detectable at the molecular level. However, the differences in molecular mechanisms take place at the macro-molecular level and may not be distinguishable at the pathway level (e.g. tetradifon and tebufenpyrad both affect the oxidative phosphorylation). The availability of high throughput molecular technologies such as microarrays and sequencing may be useful and informative techniques to elucidate such mechanisms of mixture toxicity. Indeed, through the use of these techniques, observations can be made at the level of individual genes (microarrays), at the pathway level (gene set enrichment analysis) or at the enzyme level (high throughput ELISA assays). These techniques have already been implemented in the framework of adverse outcome pathways, which can also be applied in the study of combined effects (Ankley et al., 2010). These techniques might also provide a better insight into potential similarities between the antagonistic effects observed in combinations of $M$. aeruginosa and carbaryl (Asselman et al., 2013) as well as in combinations of $M$. aeruginosa and tebufenpyrad (present study). At present, it is still unclear to what extent the antagonistic effects caused by these two combinations are similar. Indeed, these 

that are also affected by M. aeruginosa.

For the fourth insecticide, fenoxycarb, different statistical conclusions were drawn with the two different reference models (Table 1). This observation has been reported in literature and attributed to among others the different mathematical background of the reference models (Dresher and Boedeker, 1995; Jonker et al., 2005). Thus, for this specific combination, we further confirm the general agreement in literature that the concentration addition reference model provides more conservative estimates of mixture toxicity for risk assessment than reference independent action model (Altenburger et al., 1996 ;Faust and Schlolze, 2004). However, like Cedergreen et al. (2008), these findings and recommendations are not based on a greater accuracy of concentration addition compared to independent action. Therefore, from a mechanistic point of view, the current data and subsequent analysis cannot fully exclude or confirm synergistic interactions between fenoxycarb, a juvenile hormone analog, and $M$. aeruginosa as there is too little knowledge about how biological pathways are affected by these two stressors other than the primary molecular targets. At present, these two reference models are sometimes compared by determining whether the data falls into the $95 \%$ confidence interval from one model rather than the other (Dias da Silva et al., 2013). However, when both model fits are similar (such is the case for this combination, Fig. 2F and $2 \mathrm{~J}$ ) and their parameter values overlap (Table 1), again no conclusion can be made to select one model above the other. Requirements of accuracy are the primordial driver for pharmacokinetic studies or mechanistic studies, especially in human toxicology. Indeed, in those studies, the aim is to find the most accurate model for a given mixture. Based on the data in this study, both models are equally valid for these types of studies and one model cannot be selected above the other based on accuracy.

For risk assessment, however, the requirements of adequate protection of the aquatic ecosystems are more important than the requirement for an accurate mechanistic model Overall, both in this study and in the study by Asselman et al. (2013) concentration addition always provided effect predictions that are conservative from a risk assessment point of view compared to the observed effects, i.e. the predicted effects are always as large or larger than the observed effects. As a consequence, the protection of the ecosystem has a high probability of success with a conservative model such as the concentration addition model. Hence, risk assessment of mixture toxicity for 
combinations of insecticides and cyanobacteria based on concentration addition model predictions will likely result in a sufficient protection of the aquatic ecosystem. Such an assessment will be necessary in the future when climate change conditions will stimulate cyanobacterial blooms and also combined exposure to cyanobacteria and insecticides (Moe et al., 2012; Paerl and Huisman, 2009).

\section{Conclusions}

Insecticides with different modes of actionmolecular targets showed different interaction patterns when combined with Microcystis aeruginosa on the reproduction of Daphnia pulex. Two out of the four insecticides showed additive interaction patterns, whereas one showed antagonistic deviation patterns and another yielded different patterns dependent on the selected reference models. These results, together with previous results in literature, demonstrate that interactive effects cannot be generalized for insecticides targeting the same pathway and even for insecticides targeting the same enzyme. Overall, concentration addition provided more conservative predictions of effects than independent action. Furthermore, these effect predictions were always conservative compared to the observed effects leading to an adequate protection of the aquatic ecosystem when concentration addition model is used.

\section{Acknowledgements}

The authors thank N. De Saeyer, J. De Pecker, E. Beekman and S. Van Den Bulcke for the technical assistance. J. Asselman is the recipient of a PhD grant provided by the Flemish Institute for the Promotion of Scientific and Technological Research in Industry (IWT, Belgium). Funding was obtained from UGent Special Research Fund (BOF projects 01N01211) and from the Research Foundation Flanders (FWO project G.0229.09). 


\section{References}

Altenburger, R., Boedeker, W., Faust, M., Grimme, L.H., 1996. Regulations for combined effects of pollutants: Consequences from risk assessment in aquatic toxicology. Food Chem Toxicol 34, 1155-1157.

Ankley, G.T., Bennett, R.S., Erickson, R.J., Hoff, D.J., Hormung, M.W., Johnson, R.D., Mount, D.R., Nichols, J.W., Russom, C.L., Schmieder, P., Serrano, J.A., Tietge, J.E., Villeneuve, D.L., 2010. Adverse outcome pathways: A conceptual framework to support ecotoxicology research and risk assessment. Environ Toxicol Chem 29, 730-741.

Asselman, J., De Coninck, D.I.M., Glaholt, S.P., Colbourne, J.K., Janssen, C.R., Shaw, J.R., De Schamphelaere, K.A.C., 2012. Identification of pathways, gene networks and paralogous gene families in Daphnia pulex responding to exposure to the toxic cyanobacterium Microcystis aeruginosa. Environ Sci Technol 46, 84488457.

Asselman, J., Meys, J., Waegeman, W., De Baets, B., De Schamphelaere, K.A.C., 2013. Combined exposure of cyanobacteria and carbaryl results in antagonistic effects on the reproduction of Daphnia pulex. Accepted in Environ Toxicol Chem (DOI: 10.1002/etc.2296).

Bernatowicz, P., Pijanowska, J., 2011. Daphnia response to biotic stress is modified by PCBs. Ecotoxicol Environ Safe 74:711-718.

Carmichael, W., 1995. Toxic Microcystis and the environment. In: Watanabe, M., Harada, K., Carmichael, W., Fujiki, H., ed. Toxic Microcystis. Boca Raton, FL, CRC Press, pp. 1-12.

Cedergreen, N., Christensen, A.M., Kamper, A., Kudsk, P., Mathiassen, S.K., Streibig, J.C., Sorensen, H., 2008. A review of independent action compared to concentration addition as reference models for mixtures of compounds with different molecular target sites. Environ Toxicol Chem 27:1621-1632.

Cerbin, S., Kraak, M.H.S., de Voogt, P., Visser, P.M., Van Donk, E., 2010 Combined and single effects of pesticide carbaryl and toxic Microcystis aeruginosa on the life history of Daphnia pulicaria. Hydrobiol 543:129-138.

Daam, M.A., Cerejeira, M.J., Van den Brink, P.J., Brock, T.C.M., 2011. Is it possible to extrapolate results of aquatic microcosm and mesocosm experiments with pesticides between climate zones in Europe? Environ Sci Pollut Res 18:123-126.

De Laender, F., Janssen, C.R., De Schamphelaere, K.A.C., 2009. Non-simultaneous ecotoxicity testing of single chemicals and their mixture results in erroneous conclusions about the joint action of the mixture. Chemosphere 76:428-432.

Demott, W.R.; Zhang, Q.X.; Carmichael, W.W. Effects of Toxic Cyanobacteria and Purified Toxins on the Survival and Feeding of a Copepod and Three Species of Daphnia. Limnol. Oceanogr . 1991, 36, 1346-1357.

Deneer, J.W., 2000. Toxicity of mixtures of pesticides in aquatic systems. Pest Manage Sci 56:516-520.

De Zwart, D., Posthuma, L., 2009. Complex mixture toxicity for single and multiple species: Proposed methodologies. Environ Toxicol Chem 24:2665-2676.

Dias da Silva, D., Silva, E., Carvalho, F., Carmo, H., 2013. Mixtures of 3,4-methylenedioxymethamphetamine (ectasy) and its major human metabolites act additively to induce significant toxicity to liver cells when combined at low, non-cytotoxic concentrations. J Appl Toxicol, DOI: 10.1002/jat.2885

Dresher, K., Boedeker, W., 1995. Assessment of the combined effects of substances: the relationship between concentration addition and independent action. Biometrics 51, 716-730. 
Faust, M., Scholze, M. 2004 Competing concepts for the prediction of mixture toxicity: Do the differences matter for regulatory purposes? EVKI-CT-1999-00012, European Union project BEAM, final. European Commission, Brussels, Belgium.

Fox, J., 2008. Applied Regression Analysis and Generalized Linear Models, Second Edition. SAGE Publications. 665p.

Fukuto TR, 1990. Mechanism of action of organophosphorus and carbamates insecticides. Environ Health Perspect $87,245-254$.

Holmstrup, M., Bindesbøl, A.-M., Oostingh, G.J., Duschl, A., Scheil, V., Köhler, H.-R., Loureiro, S., Soares, A.M.V.M., Ferreira, A.L.G., Kienle, C., Gerhardt, A., Laskowski, R., Kramarz, P.E., Bayley, M., Svendsen, C., Spurgeon, D.J. Interactions between effects of environmental chemicals and natural stressors: A review. Sci Total Environ 408, 3746-3762.

Insecticide Resistance Action Committee (IRAC), 2009. Mode of Action Classification. Available online: http://www.irac-online.org/wp-content/uploads/2009/09/pp_moa_structure_poster_v2.7_16oct09.pdf

Kilham, S.S., Kreeger, D.A., Lynn, S.G., Goulden, C.E., Herrera, L., 1998. COMBO: a defined freshwater culture medium for algae and zooplankton. Hydrobiologia 377:147-159.

Landis, W.G., Durda, J.L., Brooks, M.L., Chapman, P.M., Menzie, C.A., Stahl, R.G. Jr, Stauber, J.L,. 2013. Ecological risk assessment in the context of global climate change. Environ Toxicol Chem 32:79-92.

Laskowski, R., Bednarska, A.J., Kramarz, P.E., Loureiro, S., Scheil, S., Kudłek, J., Holmstrup, M., 2010. Interactions between toxic chemicals and natural environmental factors - A meta-analysis and case studies. Sci Total Environ 408, 3763-3774.

Lürling, M., 2003. Daphnia growth on microcystin-producing and microcystin-free Microcystis auerginosa in different mixtures with the green alga Scenedesmus obliquus. Limnol Oceanogr 48:2214-2220.

Jöhnk, K.D., Huisman, J., Sharples, J., Sommeijer, B., Visser, P.M., Stroom, J.M., 2008. Summer heatwaves promote blooms of harmful cyanobacteria. Glob Change Biol 14:495-512.

Jonker, M.J., Svendsen, C., Bedaux, J.J.M., Bongers, M., Kammenga, J.E., 2005. Significance testing of synergistic/antagonistic, dose level-dependent, or dose ratio-dependent in mixture dose-response analysis. Environ Toxicol Chem 24:2701-2713.

Moe, S.J., De Schamphelaere, K.A.C., Clements, W.H., Sorensen, M.T., Van den Brink, P.J., Liess, M., 2012. Combined and interactive effects of global climate change and toxicants on populations and communities. Environ Toxicol Chem 32, 49-61.

OECD (Organization for Economic Cooperation and Development), 1998. Guideline for the testing of chemicals: Daphnia magna reproduction test, No. 211.

Paerl, H.W., Hall, N.S., Calandrino, E.S. 2011. Controlling harmful cyanobacterial blooms in a wolrd experiencing anthropogenic and climatic-induced change. Sci Total Environ 409, 1739-1745.

Paerl, H.W., Huisman, J., 2009. Climate change: a catalyst for global expansion of harmful cyanobacterial blooms. Environ Microbiol Rep 1:27-37.

Pope, C., Karanth, S., Liu, J. 2005. Pharmacology and toxicology of cholinesterase inhibitors: uses and misuses of a common mechanism of action. Environ Toxicol Pharmacol 19, 433-446.

R Development Core Team (2008). R: A language and environment for statistical computing. R Foundation for Statistical Computing, Vienna, Austria. ISBN 3-900051-07-0, URL http://www.R-project.org. 
453

454

455

456

457

458

459

460

461

462

463

464

465

466

467

468

469

470
Rohrlack, T., Dittmann, E., Henning, M., Börner, T., Kohl, J-G., 1999. Role of microcystin in poisoning and food ingestion inhibition of Daphnia galeata caused by the cyanobacterium Microcystis aeruginosa. Appl Environ Microbiol 65, 737-739.

Royston, P., 1982. An extension of Shapiro and Wilk's W test for normality to large samples. Applied Statistics, 31, 115-124.

Shaw, J.R., Colbourne, J.K., Davey, J.C., Glaholt, S.P., Hampton, T.H., Chen, C.Y., Folt, C.L., Hamilton, J.W., 2007. Gene response profiles for Daphnia pulex exposed to the environmental stressor cadmium reveals novel crustaceaen metallothioneins. BMC Genomics 8:477.

Shere, T.B., Richardson, J.R., Testa, C.M., Seo, B.B., Panov, A.V., Yagi, T., Matsuno-Yagi, A., Miller, G.W., Greenamyre, J.T., 2006. Mechanism of toxicity of pesticides acting at complex I: relevance to environmental etiologies of Parkinsons's disease. J Neurochem 100, 1469-1479.

Stanier, R.Y., Kunisawa, R., Mandel, M., Cohen-Bazire, G., 1971. Purification and properties of unicellular blue-green algae (order Chroococcales). Bacteriol Rev 35, 171-205.

van Apeldoorn, M.E., van Egmond, H.P., Speijers, G.J.A., Bakker, J.I., 2007. Toxins of cyanobacteria. Mol Nutr Food Res 51, 7-60.

van Gestel, C.A.M., Jonker, M., Kammenga, J.E., Laskowski, R., Svendsen, C., 2010. Mixture toxicity: Linking approaches from ecological and human toxicology. First ed., CRC Press, Florida. 


\section{Tables}

472 Table 1 Estimated model parameters and their standard error: EC50 (50\% effect concentration), s (slope parameter), and a (deviation parameter to quantify mixture interaction) for each of the different steps: IA(independent action, Eq. 3) or CA(concentration addition, Eq. 2)-model step 1 (reference model based on data from single stressors treatments only), IA or CA-model step 2 (reference model based on data from all treatments), IA or CA-model step 3 (reference model including the deviation parameter a to quantify mixture interaction, Eq.4) per cyanobacteria. The reported $p$ value is for the F-test that compared the nested models from step 2 and step 3. P $<0.05$ indicates a significant deviation from the reference model (i.e. an interactive effect).

\begin{tabular}{|c|c|c|c|c|}
\hline & Chlorpyrifos & Fenoxycarb & Tebufenpyrad & Tetradifon \\
\hline \multicolumn{5}{|l|}{ Slope parameter (s) Insecticide: } \\
\hline IA: step 1 & $1.52 \pm 0.51$ & $1.33 \pm 0.44$ & $2.60 \pm 0.57$ & $1.00 \pm 0.25$ \\
\hline IA: step2 & $1.56 \pm 0.47$ & $1.51 \pm 0.33$ & $2.70 \pm 0.52$ & $0.93 \pm 0.23$ \\
\hline IA: step3 & $2.24 \pm 0.71$ & $2.01 \pm 0.43$ & $2.25 \pm 0.50$ & $0.99 \pm 0.24$ \\
\hline CA: step1 & $1.98 \pm 0.30$ & $2.26 \pm 0.50$ & $2.11 \pm 0.37$ & $1.85 \pm 0.37$ \\
\hline CA: step2 & $3.06 \pm 0.39$ & $3.19 \pm 0.34$ & $2.86 \pm 0.61$ & $1.81 \pm 0.27$ \\
\hline CA: step3 & $3.04 \pm 0.39$ & $3.27 \pm 0.37$ & $2.16 \pm 0.38$ & $1.75 \pm 0.29$ \\
\hline \multicolumn{5}{|l|}{ Slope parameter (s) Microcystis: } \\
\hline IA: step 1 & $2.13 \pm 0.37$ & $2.98 \pm 1.02$ & $1.55 \pm 0.42$ & $2.89 \pm 0.76$ \\
\hline IA: step2 & $2.76 \pm 0.46$ & $3.44 \pm 0.63$ & $2.99 \pm 0.85$ & $2.48 \pm 0.57$ \\
\hline IA: step3 & $2.96 \pm 0.51$ & $3.71 \pm 0.64$ & $1.66 \pm 0.50$ & $2.55 \pm 0.63$ \\
\hline CA: step1 & $1.98 \pm 0.30$ & $2.26 \pm 0.50$ & $2.11 \pm 0.37$ & $1.85 \pm 0.37$ \\
\hline CA: step2 & $3.06 \pm 0.39$ & $3.19 \pm 0.34$ & $2.86 \pm 0.61$ & $1.81 \pm 0.27$ \\
\hline CA: step3 & $3.04 \pm 0.39$ & $3.27 \pm 0.37$ & $2.16 \pm 0.38$ & $1.75 \pm 0.29$ \\
\hline \multicolumn{5}{|l|}{ EC50(Insecticide) $\left(\mu \mathrm{g} \mathrm{L}^{-1}\right)$ : } \\
\hline IA: step 1 & $75.53 \pm 14.65$ & $69.37 \pm 10.16$ & $10.98 \pm 0.94$ & $11.23 \pm 2.00$ \\
\hline IA: step2 & $71.74 \pm 13.82$ & $57.80 \pm 5.17$ & $11.67 \pm 0.81$ & $9.18 \pm 1.45$ \\
\hline IA: step3 & $66.36 \pm 8.76$ & $65.97 \pm 5.03$ & $10.35 \pm 1.01$ & $10.59 \pm 1.94$ \\
\hline CA: step1 & $68.23 \pm 6.69$ & $66.09 \pm 5.66$ & $10.58 \pm 1.05$ & $12.19 \pm 1.75$ \\
\hline CA: step2 & $64.23 \pm 4.49$ & $66.44 \pm 3.15$ & $13.83 \pm 1.11$ & $10.86 \pm 1.28$ \\
\hline CA: step3 & $62.48 \pm 4.56$ & $65.41 \pm 3.32$ & $10.37 \pm 1.03$ & $11.55 \pm 1.56$ \\
\hline \multicolumn{5}{|l|}{ EC50(Microcystis) (\% of diet): } \\
\hline IA: step 1 & $37.61 \pm 3.56$ & $28.74 \pm 3.93$ & $55.40 \pm 9.51$ & $30.48 \pm 3.13$ \\
\hline IA: step2 & $32.75 \pm 1.89$ & $25.55 \pm 1.35$ & $64.11 \pm 7.41$ & 31.512 .83 \\
\hline IA: step3 & $36.42 \pm 2.94$ & $29.37 \pm 1.90$ & $58.30 \pm 9.96$ & $33.17 \pm 3.50$ \\
\hline CA: step1 & $37.66 \pm 3.67$ & $30.04 \pm 4.59$ & $54.62 \pm 7.65$ & $33.61 \pm 5.49$ \\
\hline CA: step2 & $41.06 \pm 2.34$ & $30.76 \pm 1.78$ & $88.07 \pm 12.45$ & $32.87 \pm 3.92$ \\
\hline CA: step3 & $37.97 \pm 3.17$ & $29.58 \pm 2.33$ & $57.74 \pm 8.30$ & $34.54 \pm 5.27$ \\
\hline \multicolumn{5}{|l|}{ a: } \\
\hline IA: step3 & $-1.51 \pm 0.86$ & $-2.10 \pm 0.63$ & $2.37 \pm 0.88$ & $-0.74 \pm 0.67$ \\
\hline CA: step3 & $0.37 \pm 0.33$ & $0.23 \pm 0.32$ & $2.703 \pm 0.64$ & $-0.44 \pm 0.64$ \\
\hline Conclusion IA: & Additivity & Synergism & Antagonism & Additivity \\
\hline P-value (IA: step2/IA: step3) & 0.1035 & 0.001584 & 0.03555 & 0.2813 \\
\hline Conclusion CA: & Additivity & Additivity & Antagonism & Additivity \\
\hline P-value (CA: step2/CA: step3) & 0.2853 & 0.4851 & $<0.00001$ & 0.4793 \\
\hline
\end{tabular}




\section{Figure Captions}

480 Figure 1. Experimental designs for the binary mixture combinations: Nominal concentrations are represented by 481 filled circles, measured concentrations are represented by open squares with error bars representing standard 482 deviation. Control treatment is represented by an open circle. A: Chlorpyrifos $\times$ M. aeruginosa. B: Fenoxycarb $\times$ M. 483 aeruginosa. C: Tebufenpyrad x M. aeruginosa. D: Tetradifon x M. aeruginosa.

Figure 2. Mean observed versus fitted values for models for total reproduction for each binary experiment: chlorpyrifos $\mathrm{x}$ M. aeruginosa (first row), fenoxycarb $\mathrm{x}$ M. aeruginosa (second row), tebufenpyrad $\mathrm{x}$ M. aeruginosa (third row), tetradifon $\times$ M. aeruginosa (fourth row). Circles depict the independent action model fits (Eq. 3), triangles depict the concentration addition model fits (Eq. 2). Open symbols denote the single stressor treatments,

489 full symbols denote the mixed stressor treatments. Left: reference model fitted based on single stressor treatments 490 only and predicted mixture points with this fitted model, i.e. step1. Middle: reference model fitted based on all 491 data (single stressor treatment data and mixture data) i.e. step2. . Right: reference model with deviation parameter 492 'a' fitted (eq. 4) based on all data (single stressor treatment data and mixture data), i.e. step3 . For all models, the 493 1:1 line is plotted. The same type of Figures, but showing all individual replicates instead of means per treatment, 494 are visualized in Fig. S14. 
$\square$

口

ㅁ

ㅁ

口

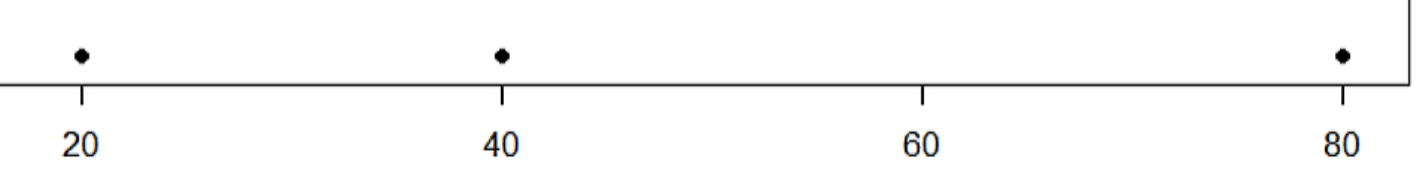

Cyanobacteria (\% of diet)

$\square$

ㅁ

口

$\square$



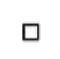

$\square$

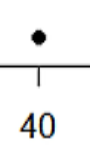

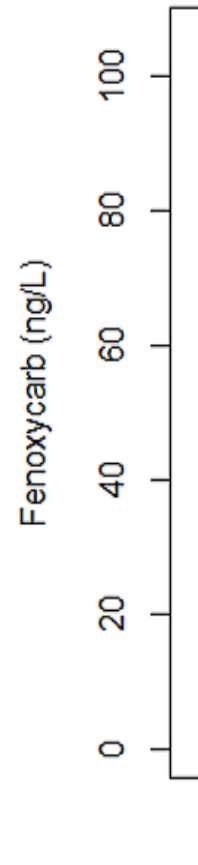
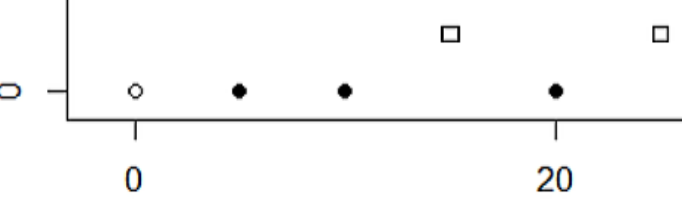

$\square$

ㅁ

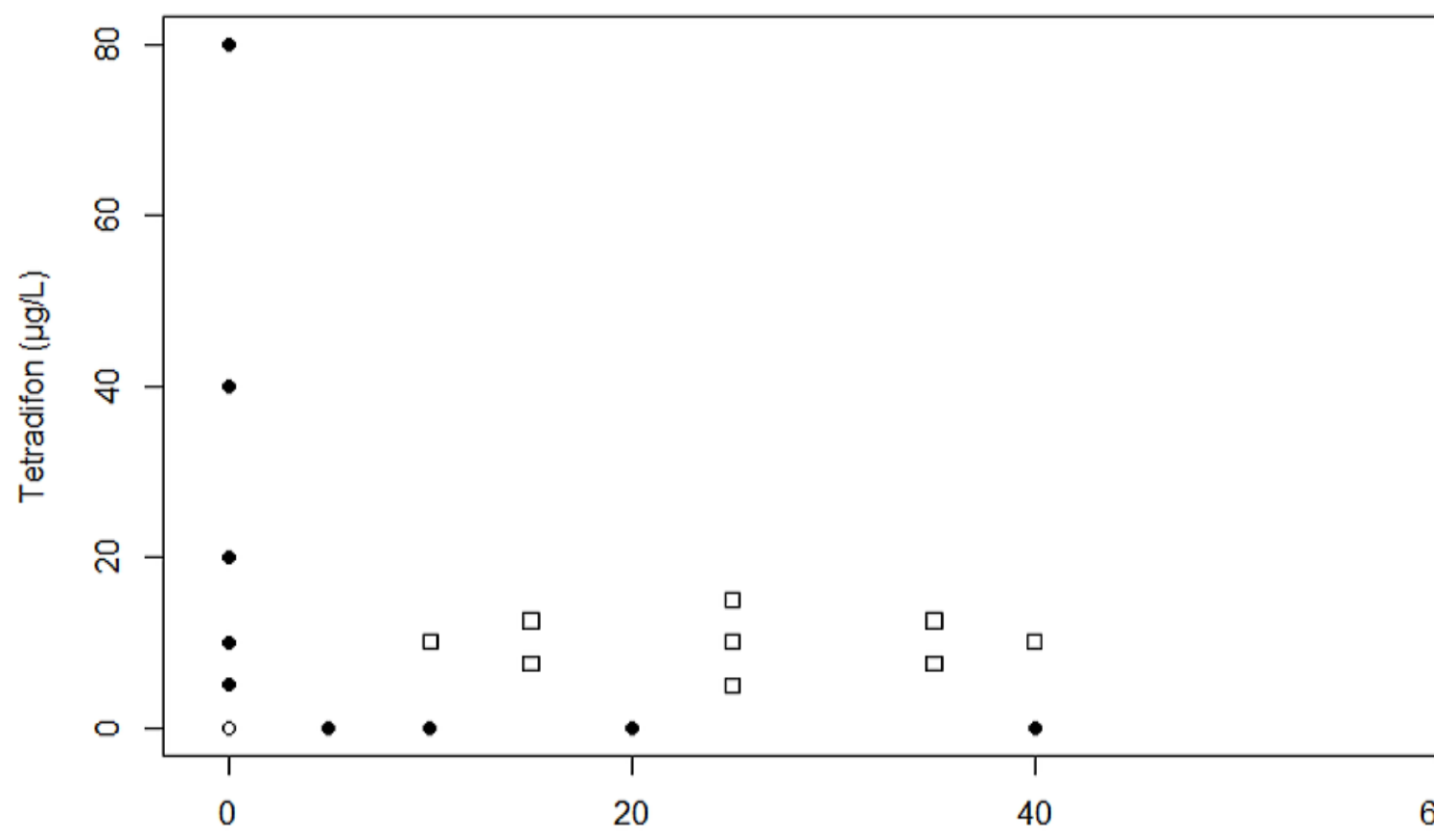

Cyanobacteria ( $\%$ of diet) 


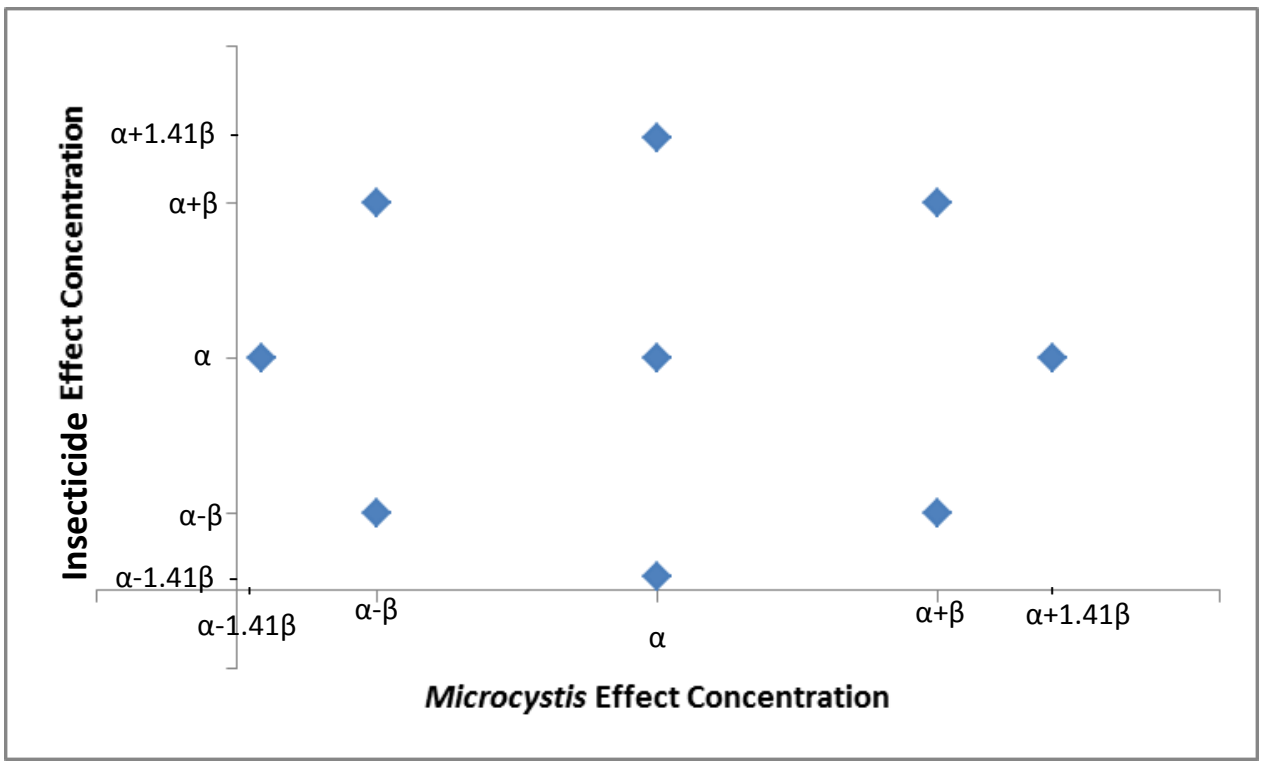

Figure S1 Central composite design adapted from Lock and Janssen (2002). $\alpha=E C 50 / 2$ whereas $\beta=E C 50 / 2-E C 10 / 2$. EC50 is the effect concentration causing $50 \%$ decline in the monitored endpoint compared to control treatments. EC10 is the effect concentration causing $10 \%$ decline in the monitored endpoint compared to control treatments.

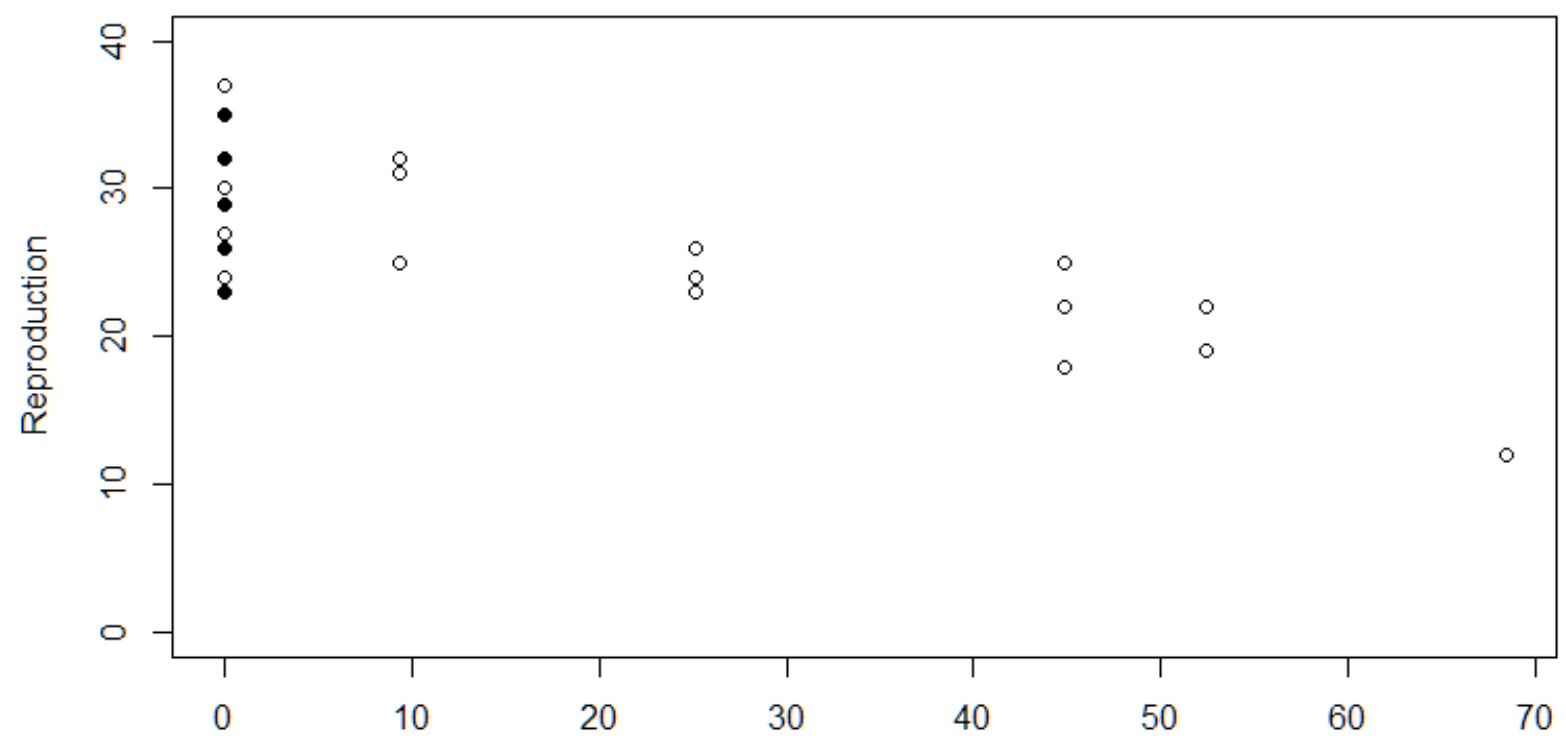

Chlorpyrifos (ng/L)

Figure S2 Dose response data for exposure to different concentrations of chlorpyrifos. Each circle represents the total reproduction of a single replicate surviving the 21 days experiment. Open circles denote all treatments that contained the solvent carrier. Filled circles represent control treatment without solvent carrier. 


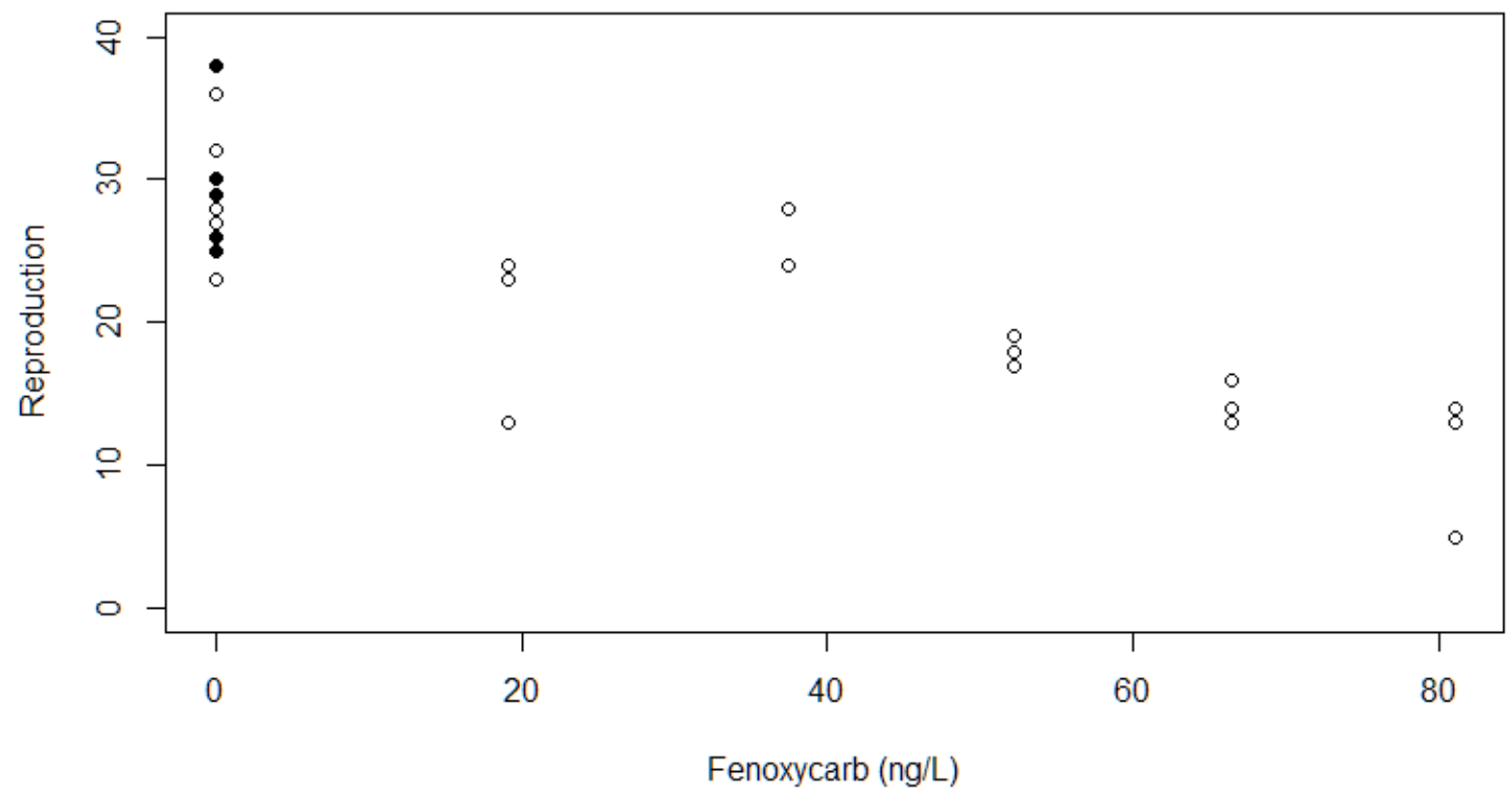

Figure S3 Dose response data for exposure to different concentrations of fenoxycarb. Each circle represents the total reproduction of a single replicate surviving the $\mathbf{2 1}$ days experiment. Open circles denote all treatments that contained the solvent carrier. Filled circles represent control treatment without solvent carrier.

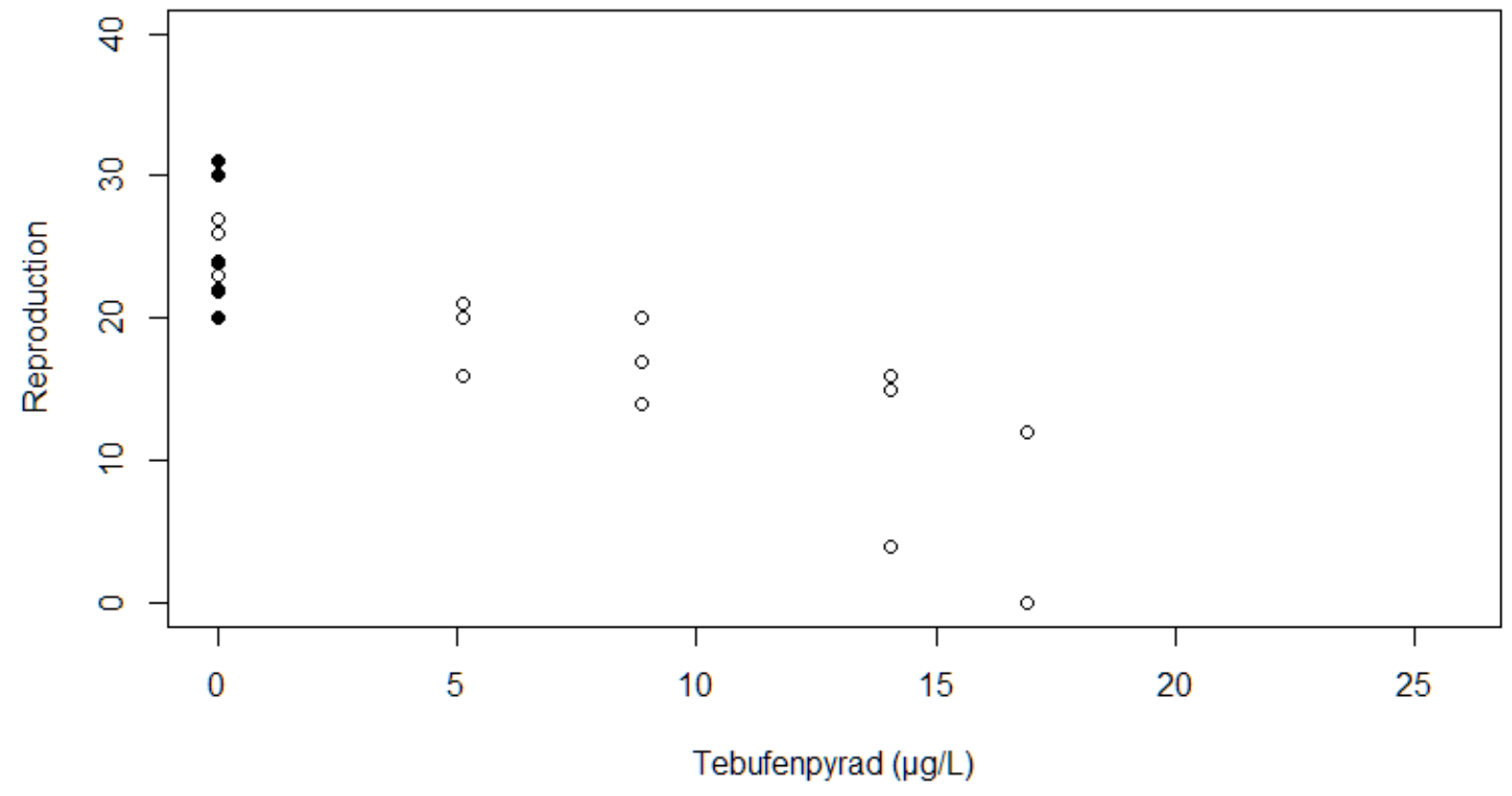

Figure S4 Dose response data for exposure to different concentrations of tebufenpyrad. Each circle represents the total reproduction of a single replicate surviving the 21 days experiment. Open circles denote all treatments that contained the solvent carrier. Filled circles represent control treatment without solvent carrier. 


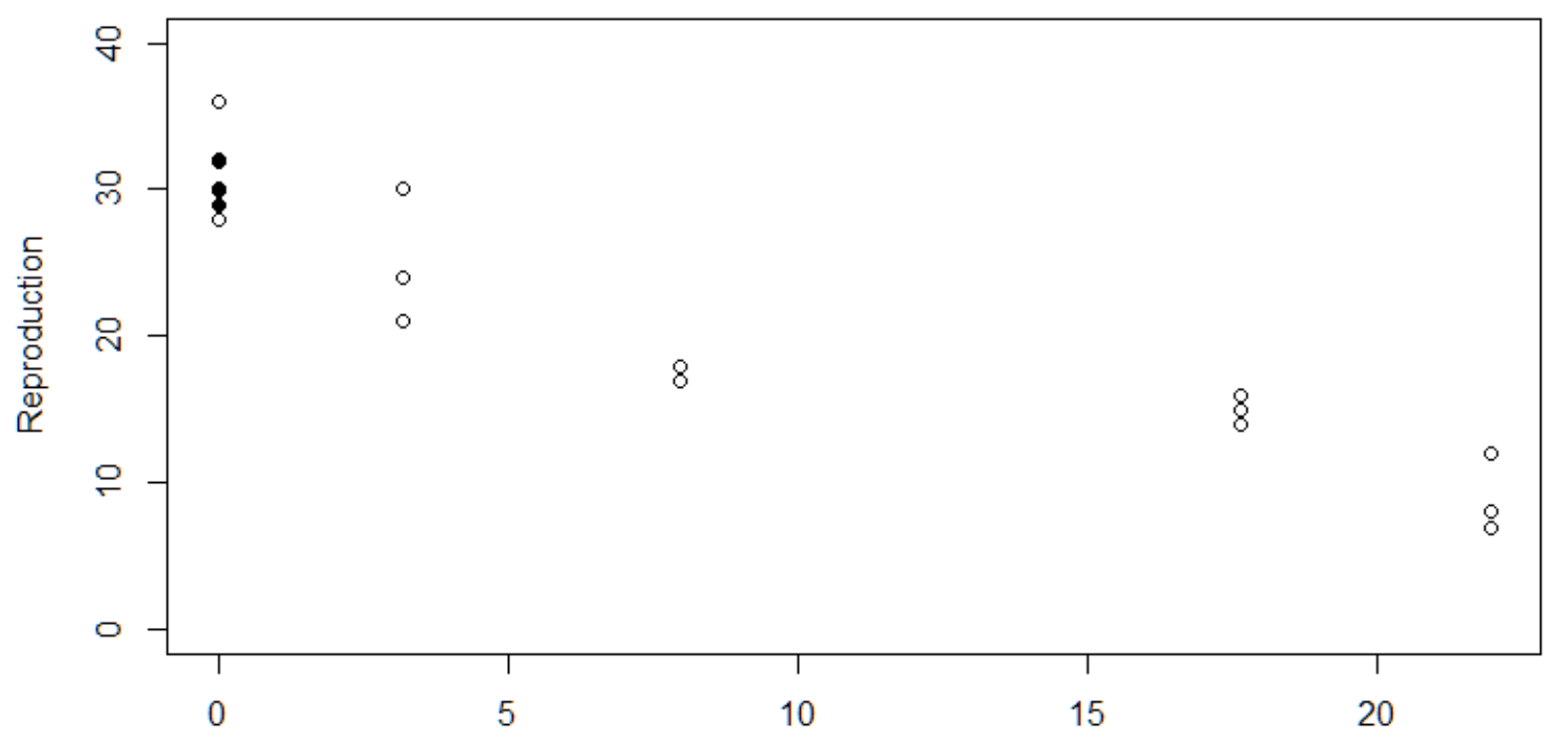

Tetradifon $(\mu \mathrm{g} / \mathrm{L})$

Figure S5 Dose response data for exposure to different concentrations of tetradifon. Each circle represents the total reproduction of a single replicate surviving the 21 days experiment. Open circles denote all treatments that contained the solvent carrier. Filled circles represent control treatment without solvent carrier.

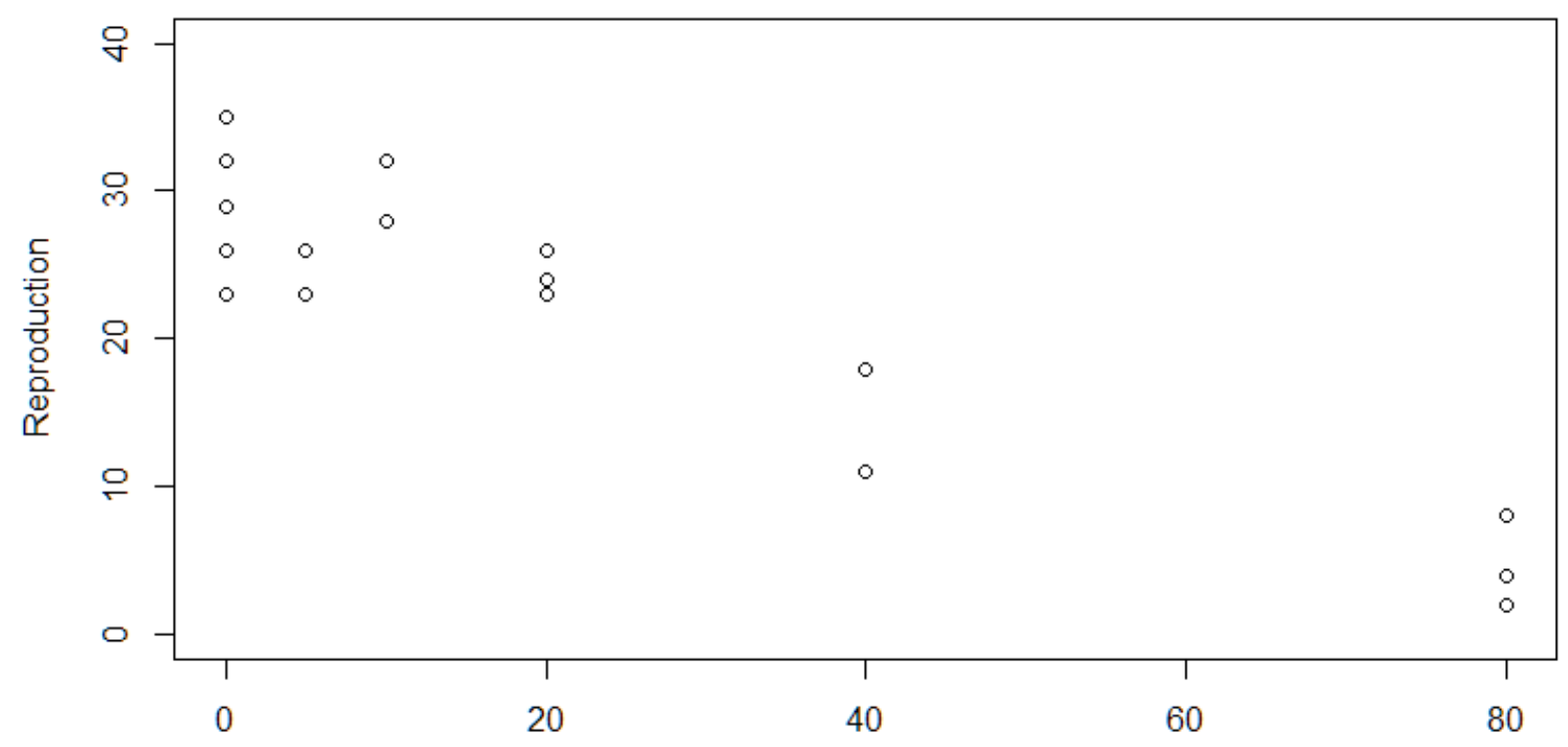

Microcystis \% of diet

Figure S6 Dose response data for exposure to different concentrations of the cyanobacteria, Microcystis aeruginosa, in the diet. Each circle represents the total reproduction of a single replicate surviving the 21 days experiment. 


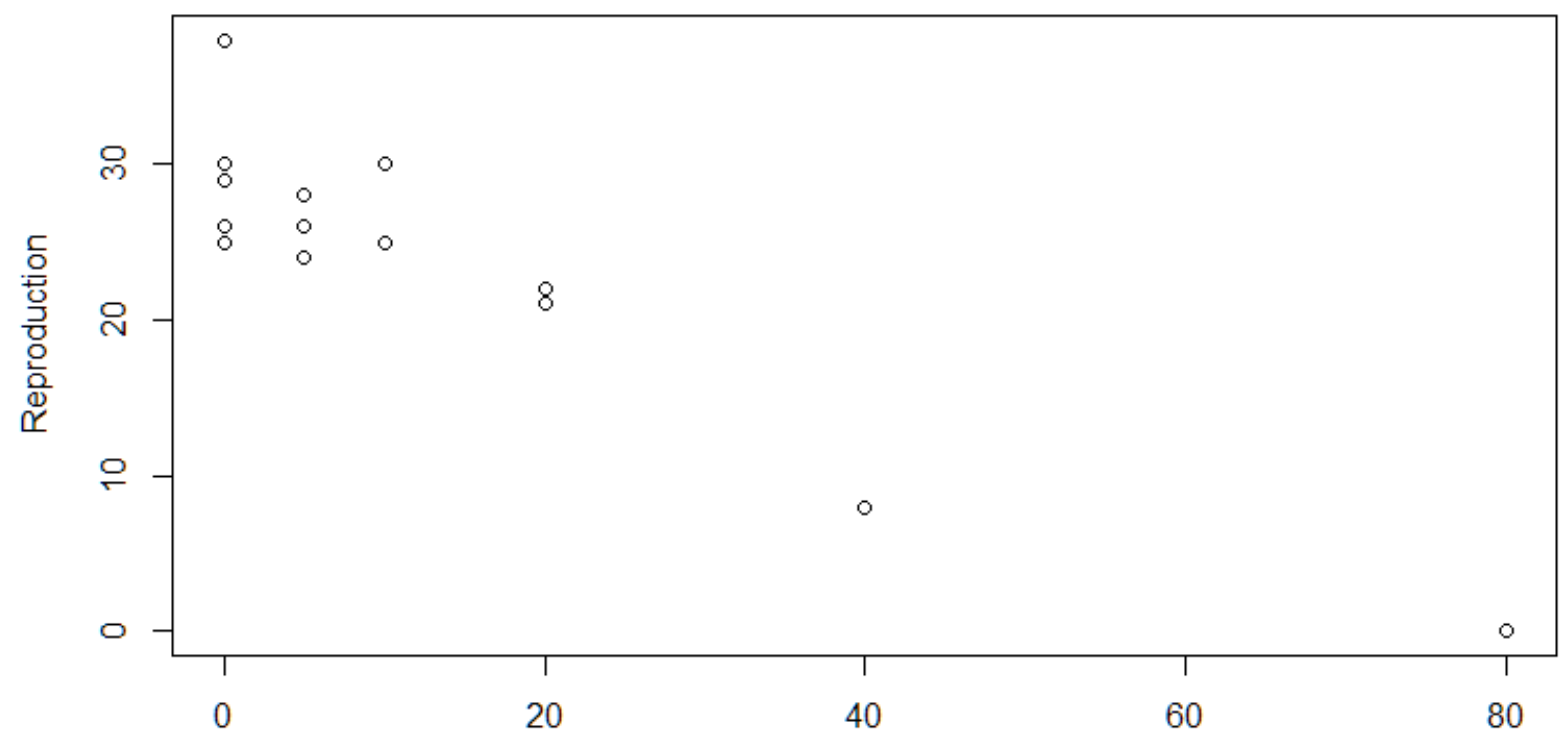

Microcystis \% of diet

Figure S7 Dose response data for exposure to different concentrations of the cyanobacteria, Microcystis aeruginosa, in the diet. Each circle represents the total reproduction of a single replicate surviving the $\mathbf{2 1}$ days experiment.

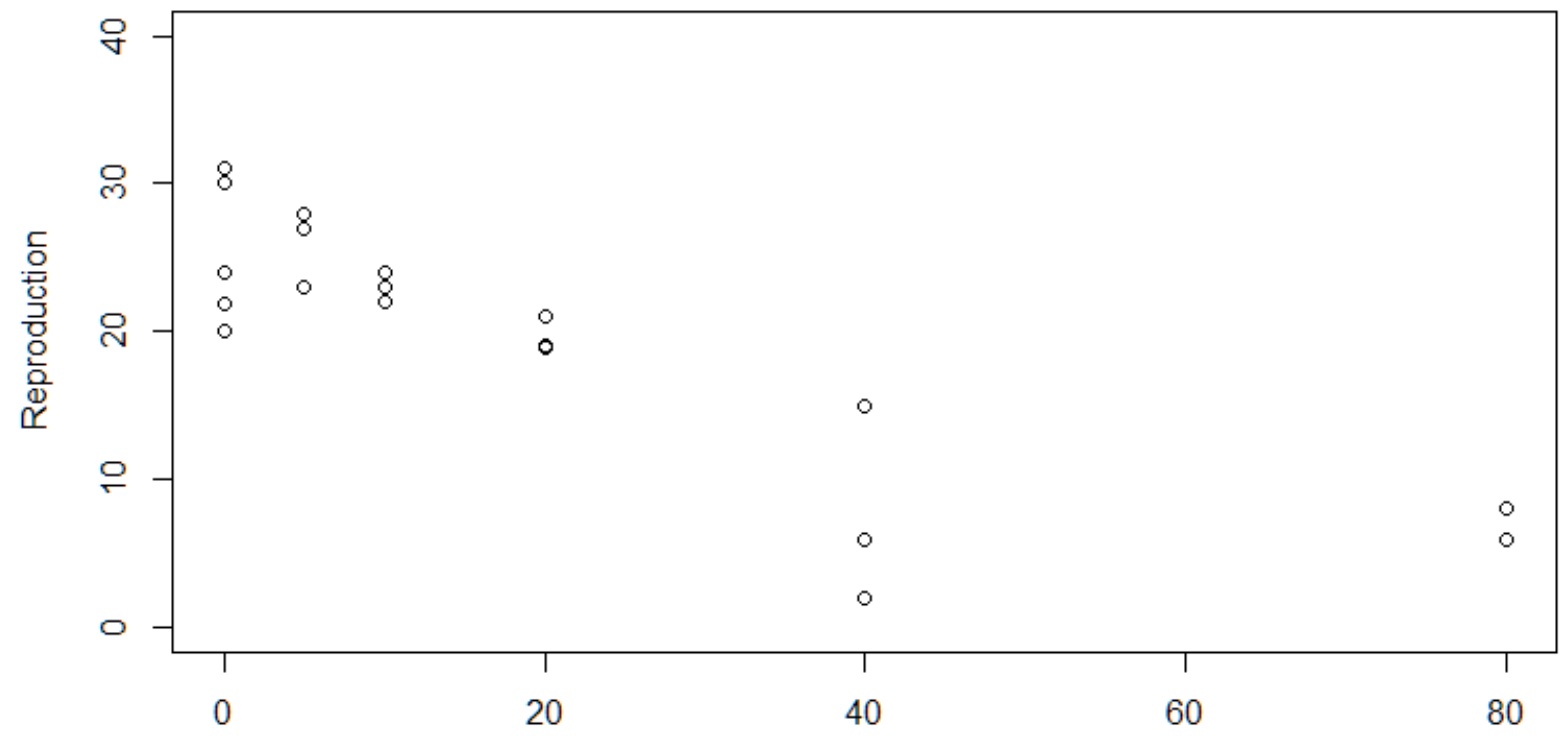

Microcystis \% of diet

Figure S8 Dose response data for exposure to different concentrations of the cyanobacteria, Microcystis aeruginosa, in the diet. Each circle represents the total reproduction of a single replicate surviving the $\mathbf{2 1}$ days experiment. 


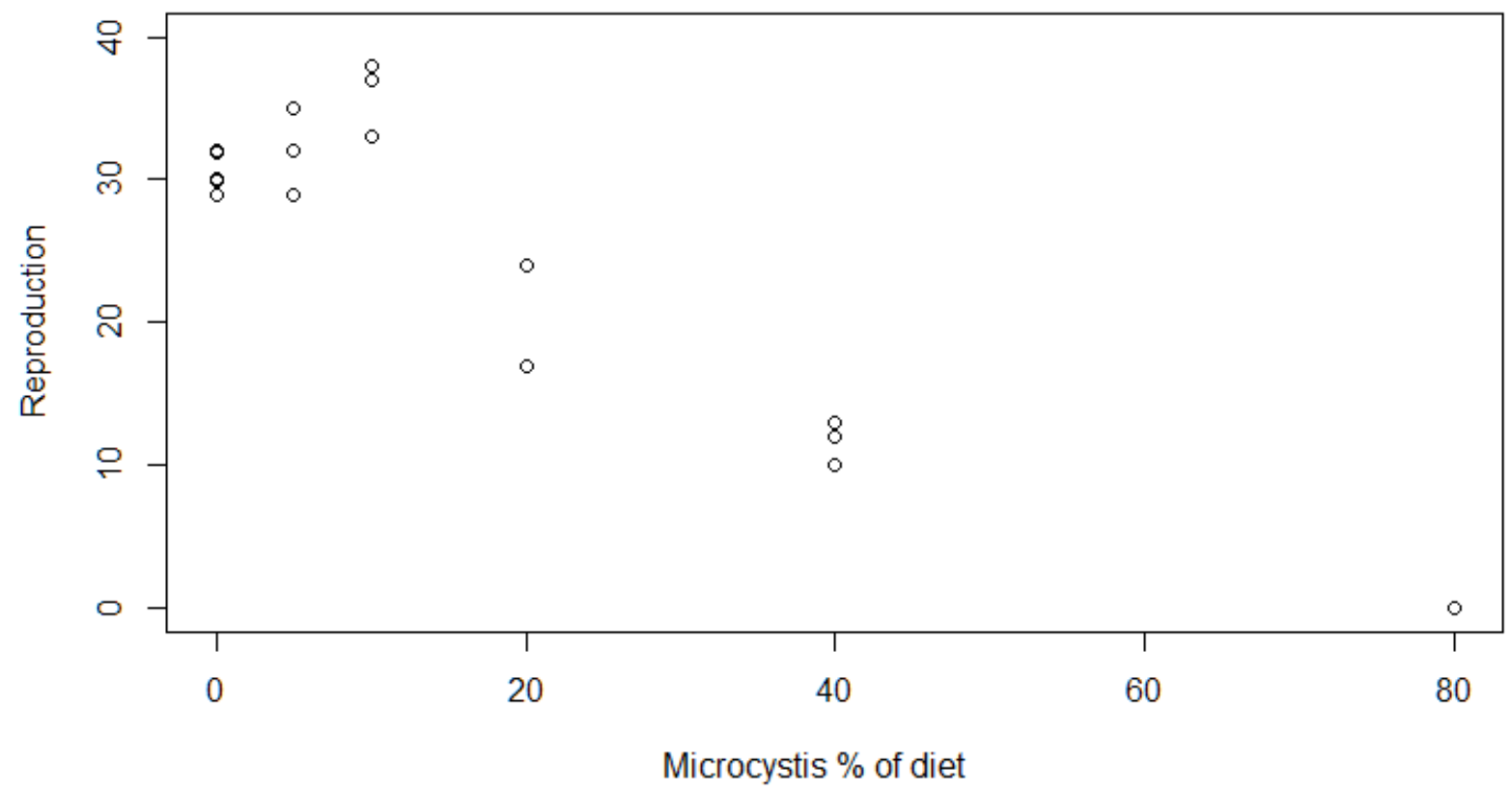

Figure S9 Dose response data for exposure to different concentrations of the cyanobacteria, Microcystis aeruginosa, in the diet. Each circle represents the total reproduction of a single replicate surviving the $\mathbf{2 1}$ days experiment. 


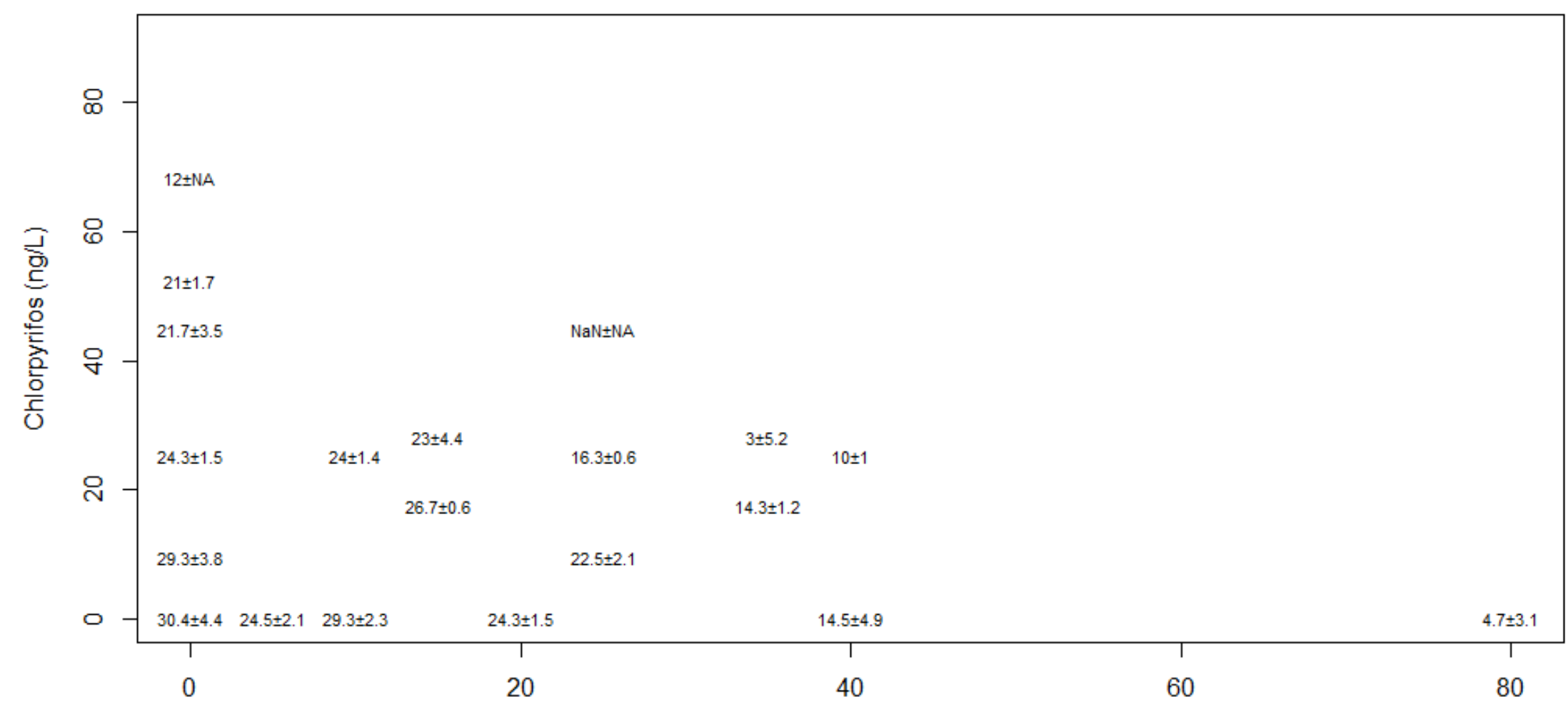

Microcystis \% of diet

Figure S10 Mean total reproduction for the surviving animal and the corresponding standard deviation per treatment. NaN means no animals survived the treatment. NA means no standard deviation could be computed due to only one surviving replicate for that treatment. 


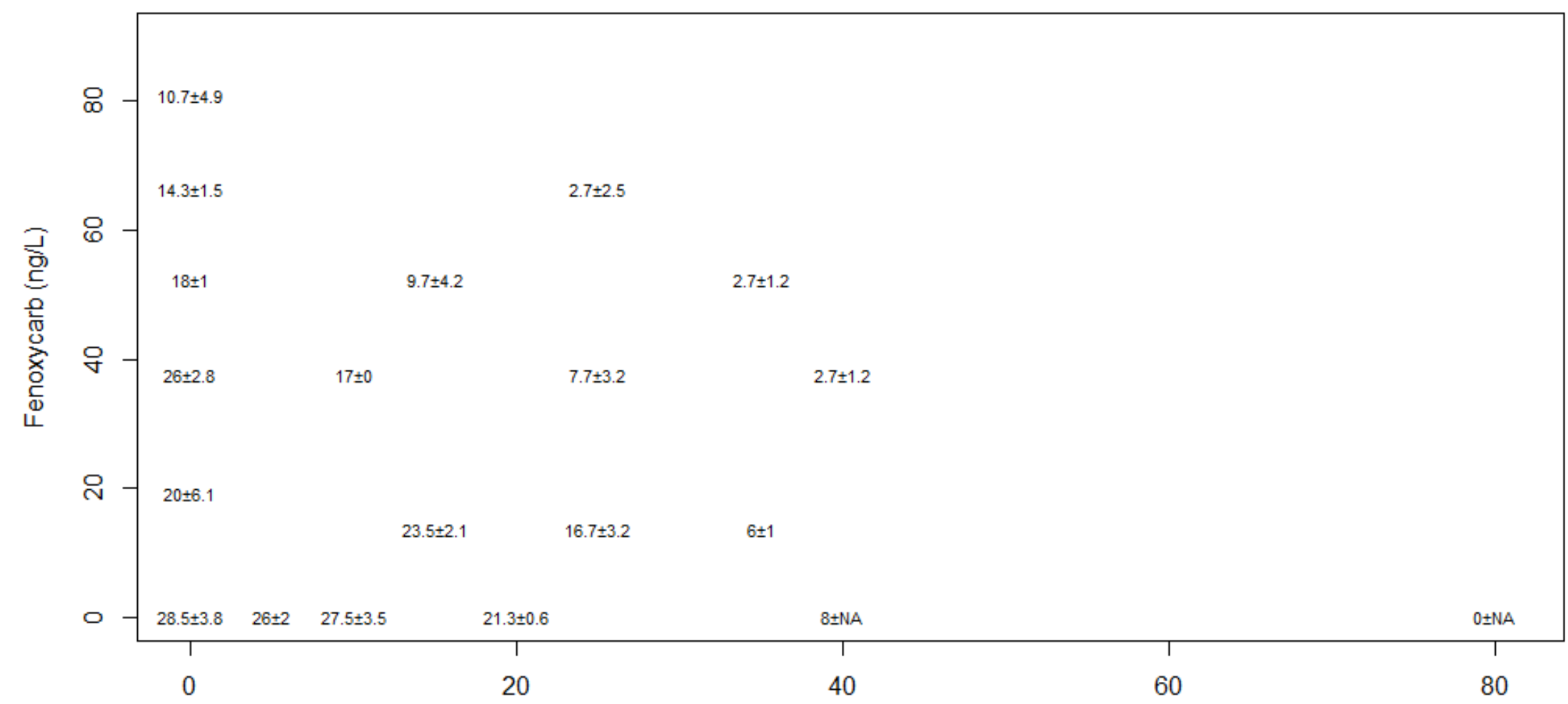

Microcystis \% of diet

Figure S11 Mean total reproduction for the surviving animal and the corresponding standard deviation per treatment. NA means no standard deviation could be computed due to only one surviving replicate for that treatment. 


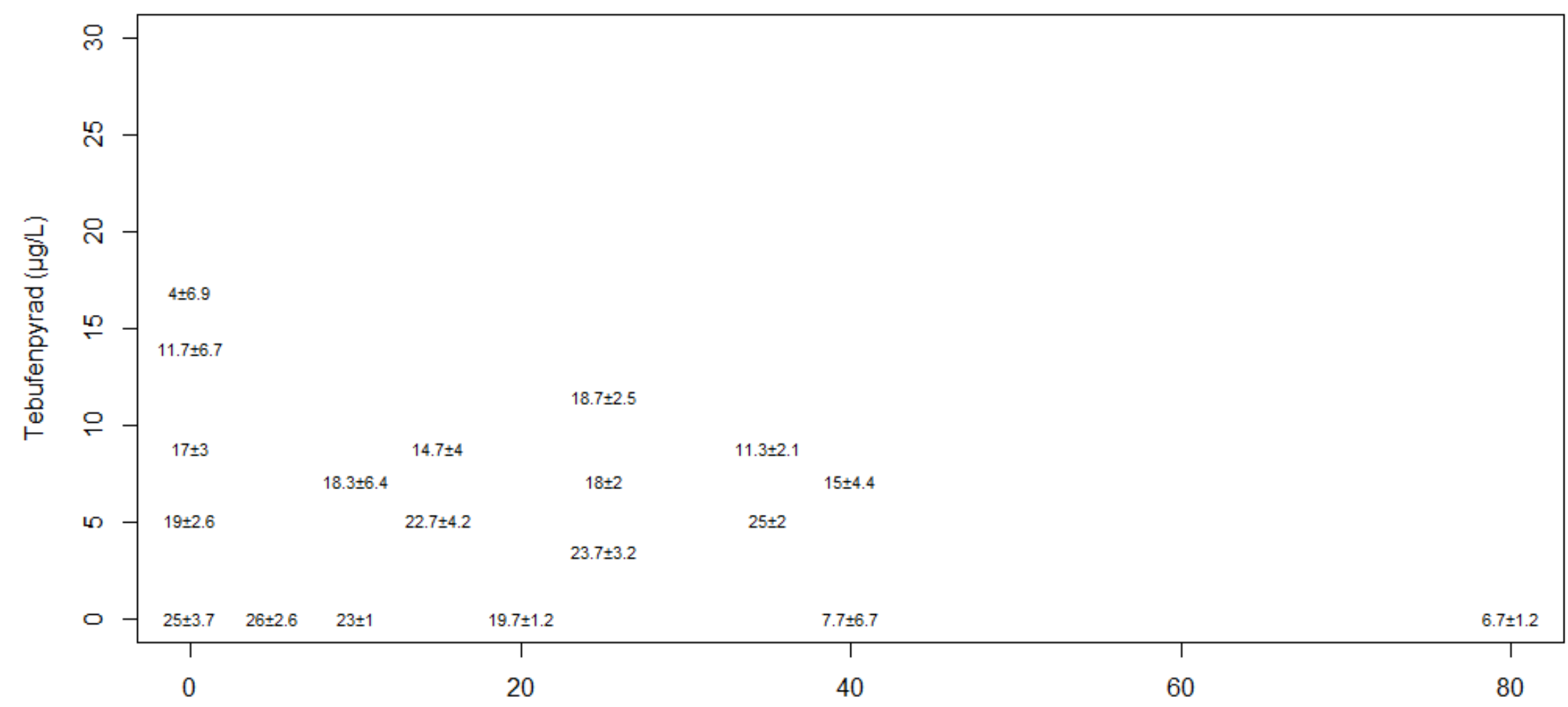

Microcystis \% of diet

Figure S12 Mean total reproduction for the surviving animal and the corresponding standard deviation per treatment. 


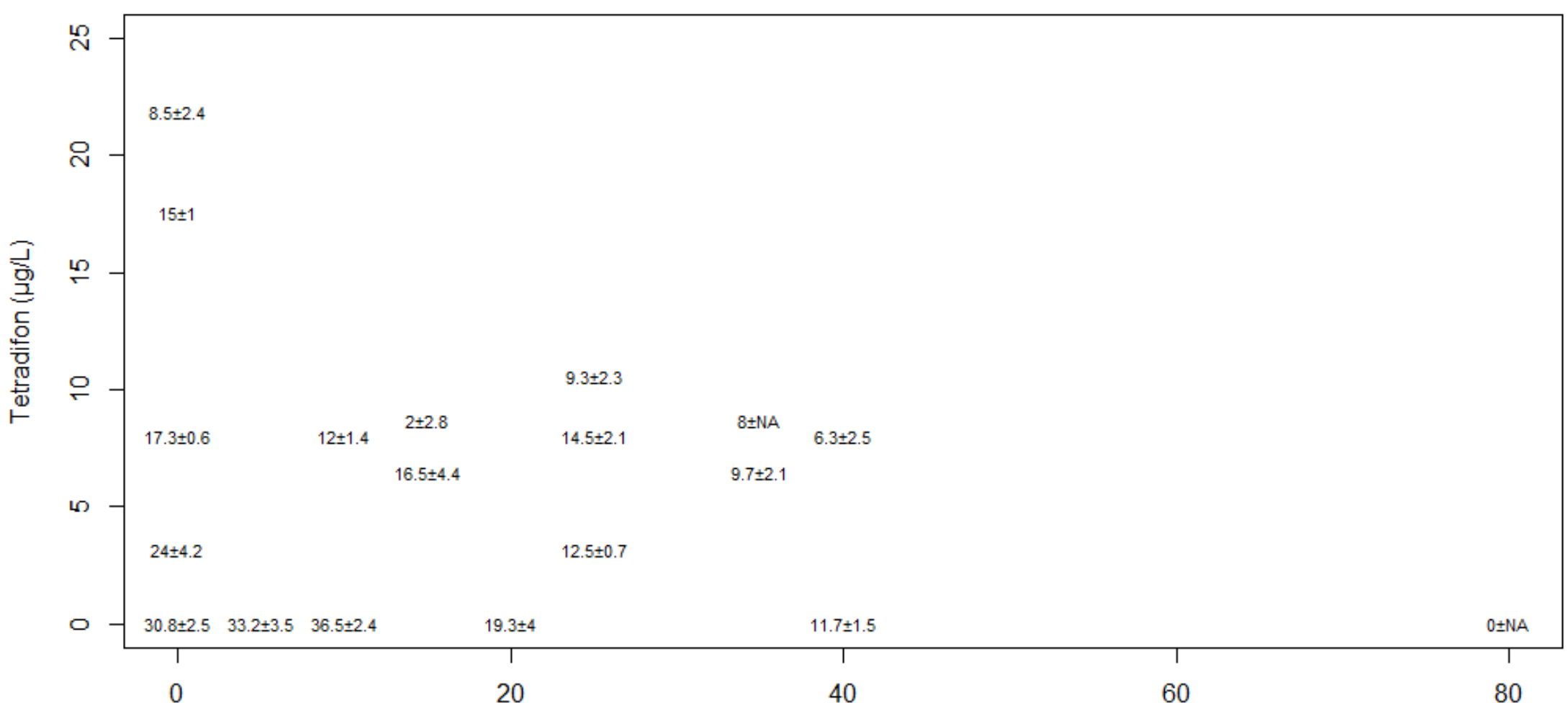

Microcystis \% of diet

Figure S13 Mean total reproduction for the surviving animal and the corresponding standard deviation per treatment. NA means no standard deviation could be computed due to only one surviving replicate for that treatment. 


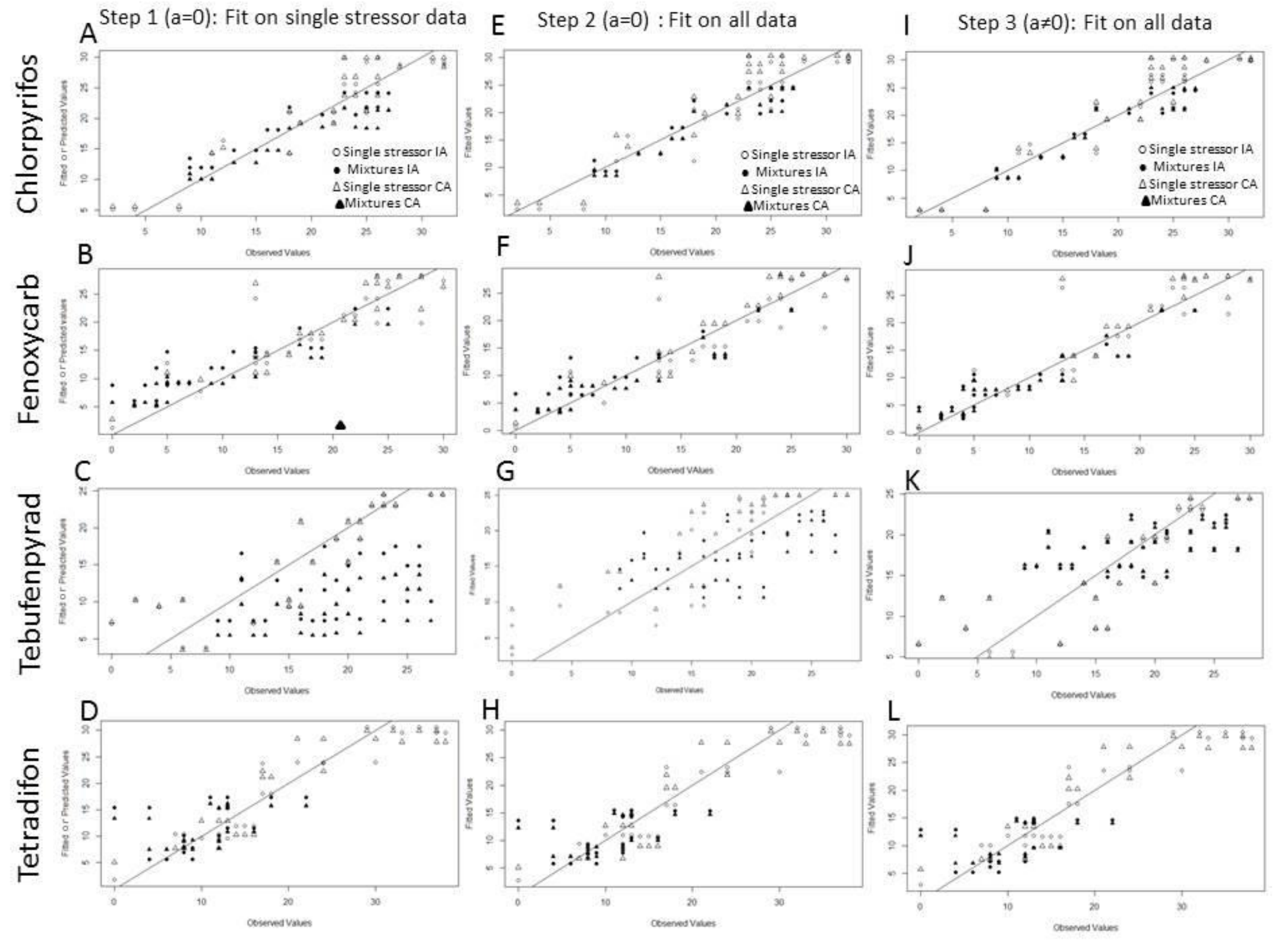


Figure S14 Observed versus predicted or fitted values for models for total reproduction for each binary experiment: chlorpyrifos x M. aeruginosa (first row), fenoxycarb x M. aeruginosa (second row), tebufenpyrad x $M$. aeruginosa (third row), tetradifon $\times$ M. aeruginosa (fourth row). Circles depict the independent action model fits, triangles depict the concentration addition model fits. Left: step1, i.e. reference model based on single treatments (open symbols) and predicted mixture points (full symbols). Middle: step 2, i.e. reference model based on all data, single treatment data (open symbols) and mixture data (full symbols). Right: step 3, i.e. reference model with deviation parameter based on all data, single treatment data (open symbols) and mixture data (full symbols). For all models, the 1:1 line is plotted.

Table S1 P-values for concentration addition (CA)/ independent action (IA) when leaving one design point out. Insecticide concentrations are represented as effect concentrations (EC) based upon the general central composite design in Fig. S1. Cyanobacteria concentrations are given in \% of the diet as represented in Fig. 1 A-D. $\alpha=E C 50 / 2$ whereas $\beta=E C 50 / 2-E C 10 / 2$. EC50 is the effect concentration causing $50 \%$ decline in the monitored endpoint compared to control treatments. EC10 is the effect concentration causing $10 \%$ decline in the monitored endpoint compared to control treatments.

\begin{tabular}{llllll}
\hline Design points & \multicolumn{4}{c}{-values } \\
\hline Insecticide EC (Fig. S1) & Cyano (\% of diet) (Fig.1-4) & Chlorpyrifos & Fenoxycarb & Tebufenpyrad & Tetradifon \\
$\alpha-1.41 \beta$ & 25 & $0.136 / 0.266$ & $0.488 / 0.003$ & $<0.001 /<0.001$ & $0.312 / 0.560$ \\
$\alpha-\beta$ & 15 & $0.104 / 0.430$ & $0.638 / 0.002$ & $<0.001 /<0.001$ & $0.314 / 0.461$ \\
$\alpha-\beta$ & 35 & $0.099 / 0.298$ & $0.663 / 0.002$ & $0.001 /<0.001$ & $0.211 / 0.449$ \\
$\alpha$ & 10 & $0.115 / 0.271$ & $0.435 / 0.003$ & $<0.001 /<0.001$ & $0.378 / 0.590$ \\
$\alpha$ & 25 & $0.131 / 0.363$ & $0.487 / 0.003$ & $<0.001 /<0.001$ & $0.158 / 0.318$ \\
$\alpha$ & 40 & $0.087 / 0.342$ & $0.503 / 0.002$ & $<0.001 /<0.001$ & $0.263 / 0.512$ \\
$\alpha+\beta$ & 15 & $0.064 / 0.564$ & $0.542 / 0.009$ & $<0.001 /<0.001$ & $0.788 / 0.963$ \\
$\alpha+\beta$ & 35 & $0.603 / 0.135$ & $0.491 / 0.003$ & $<0.001 /<0.001$ & $0.281 / 0.479$ \\
$\alpha+1.41 \beta$ & 25 & $0.103 / 0.285$ & $0.498 / 0.003$ & $<0.001 /<0.001$ & $0.267 / 0.439$ \\
\hline
\end{tabular}

